\title{
Theory of Flow-Induced Fibril Formation in Polymer Solutions
}

\author{
John D. Hoffman \\ National Measurement Laboratory, National Bureau of Standards, Washington, DC 20234
}

May 7, 1979

\begin{abstract}
A treatment of the formation of a basic core fibril (shish) of the type that is generated by flow-induced crystallization of a polymer from solution is given that features the concept of cumulative strain. Multiple nucleation acts by flow-elongated molecules produce an embryonic fibril that is a connected set of bundlelike nuclei. Surface stress resulting from repulsion of the quasi-random coil chains in the amorphous zone between the nuclei or crystallites builds up at the bundle ends as the nuclei mature, leading ultimately to a high end surface free energy, and to volume strain in the crystallites comprising the core fibril. The theory leads to a stable (or metastable) fibril diameter $a_{s}$ and mean characteristic length $l_{s}$ with a fixed axial ratio, and predicts why the diameter does not grow further even in a medium that is supersaturated with polymer. The predicted dependence of $a_{s}, l_{s}$, and the axial ratio, on undercooling is in approximate agreement with experiment. The lattice expansion in the crystal resulting from volume strain is also in fair accord with experiment. The effect of annealing, including the commonly encountered case where the volume strain relaxes to give normal lattice dimensions, but with a high end surface energy still remaining, is noted. The effect of volume strain and the distribution of core fibril lengths about $l_{s}$ on the melting behavior is calculated. The theory can reproduce crystallinity versus temperature data on polyethylene fibrils. This procedure yields an independent value of $l_{s}$. The overall treatment implies that the core fibril is a set of concatenated and substantially extended-chain crystallites with bundlelike ends and a somewhat expanded lattice when unannealed and under tension, the molecular connections between the crystallites consisting of short amorphous ciliary bridges. It is suggested that prolonged annealing at high temperatures can remove a substantial number of the amorphous zones.
\end{abstract}

Key words: Core fibril; cumulative stress; flow-induced crystallization; nucleation theory; polyethylene; polymer fiber; shish; volume strain.

\section{Introduction}

Some time ago, it was discovered that very long and thin fibrils of polyethylene could be formed by rapidly stirring a solution of the polymer in a subcooled state. This type of crystallization has been systematically investigated by Pennings and coworkers [1-6]. ${ }^{1}$ These fibrils are typically a few hundred $\AA$ in diameter $(1 \AA=10 \mathrm{~nm})$ and the overall length is essentially unlimited. An interesting feature of these fibrils is that they are usually decorated at intervals by lamellar platelets. These platelet overgrowths are usually considered to be chain-folded. The combined structure is commonly called a "shish-kebab," the "shish" representing the thin central filament, and the "kebabs" the lamellar platelets that appear along the filament. A variety of related techniques (e.g., Poiseuille flow [7], Couette flow [8], and impinging jets $[9,10])$ lead to the "shish-kebab" structures in subcooled solutions of polyethylene: The common feature of the various

\footnotetext{
${ }^{1}$ Figures in brackets indicate the literature references at the end of this paper.
}

methods of preparation is that they all involve some mechanism, arising from a flow field, that has the capacity to statistically elongate some of the polymer molecules in the solution which then act as nuclei for crystallization.

Based on their work on the contraction of fibrils of polyethylene on warming, Grubb and Keller [11] have pictured the core fibril itself as a set of long thin crystallites interspersed by much shorter disordered regions. In the fibrils they studied, the melting phenomena suggested that the distribution of crystallite lengths was quite broad and asymmetric, but the mean length of the crystallites was nevertheless a well-defined quantity. (Subsequently, we shall refer to the mean length of the crystallites in the core fibril as the "characteristic length"). Grubb and Keller estimated that the length of the crystallites in their specimens was 1500 to $2000 \AA$, for fibrils having a diameter of about $300 \AA$.

No general treatment of the formation of these core fibrils appears to be available. The present paper represents an attempt in this direction, with emphasis on the prediction of certain properties that derive from the somewhat unusual conditions that prevail during the formation of these interest- 
ing systems. The treatment involves deductions from statistical mechanics, but its major elements arise from a combination of continuum mechanics and nucleation theory.

The author has for some time been intrigued by a curious feature of the central filament, namely, that it does not ordinarily seem to grow beyond a certain diameter even though it is immersed in a subcooled medium that is in effect supersaturated with polymer molecules capable of crystallization. This statement holds no matter whether the flow field is present or not. What generally happens in a quiescent nutrient medium is that the chain-folded platelets grow or even rearrange, while the core fibril does not increase in diameter. Mackley has previously noted the apparent incapacity of the core filament in a given experiment to grow beyond a certain diameter during the formation process [10]. This situation concerning the central filament appeared to the author to suggest that the fibril diameter (and possibly the characteristic length of the crystallites forming the overall core fibril) is intrinsically limited in some fashion. In the present paper the hypothesis that this limitation is a result of cumulative stresses arising from mutual repulsion of chains in the amorphous regions is explored.

In specimens prepared from solution, the lamellar platelets occur at intervals of varying regularity along the core fibril. The question then arises as to what connection there is if any between the mean interplatelet distance, and the mean length of the crystallite comprising the core fibril, i.e., the characteristic length as previously defined. In our analysis of the data given by George and Tucker [12] on specially prepared specimens where excessive additional overgrowths were minimized by appropriate techniques, we shall tentatively assume that these two quantities are approximately the same. George and Tucker assumed that the lamellar overgrowths actually occurred at the crystallite ends. This assumption was evidently based on the idea that cilia emanating from the discontinuities at the crystallite ends provided material that initiated the lamellar overgrowths. There is in fact a quite different assumption that also leads to a close connection between the mean value of the distance between the lamellar platelets and the characteristic length, and this will be noted later. The assumption that the mean value of the interplatelet distance is similar to the mean value of the crystallite length in suitable preparations is subject to some question, since Pennings and coworkers have recently shown that different interplatelet spacings can be produced on the same core fibril, and that after prolonged storage the spacing is a function of this storage temperature [13]. However, this does not necessarily imply that the mean distance between the lamellar overgrowths in a fresh preparation where care has been taken to minimize extra overgrowths is totally unrelated to the mean crystallite length in the core fibril. This question will be dealt with further, and is mentioned here mainly for the purpose of indicating at the outset that there is some uncertainty in the estimates of the crystallite length in certain experiments that will be analyzed in terms of the theory.

For polyethylene crystallized from $n$-octadecane under shear, it has been found by George and Tucker that both the diameter of the fibrils and the mean lamellar overgrowth spacing are dependent on the temperature of crystallization, the greater diameters and interlamellar spacings being associated with the higher crystallization temperature. The mean value of the interlamellar spacings is typically about seven to eight times larger than the fibril diameter. (Results obtained by other investigators to be noted subsequently suggest a ratio close to six.) George and Tucker also found by electron diffraction experiments that the lateral spacings of the polyethylene molecules in the orthorhombic lattice of their specimens were decidedly larger than normal; the crosssectional area occupied by the chains in fibrils $181 \AA$ in diameter was expanded by circa 4.7 percent. This is consistent with the concept that, under certain circumstances, volume strain can exist in the fibrils.

It will emerge that the application of the concept of cumulative volume strain originating at the bundle ends of a fringed-micellar type of crystallite can lead to a stable (or metastable) diameter for the fundamental units that comprise the core filament, and thus explain the limited radial growth of these entities. The fibril diameter is predicted to vary as the reciprocal of the undercooling. A similar limitation is found for the characteristic length, which is also predicted to vary as the reciprocal of the undercooling. The results are such that the crystallites making up the core fibril possess a constant mean axial ratio at various undercoolings, and a simple rationale is provided for the absolute value of the axial ratio. Insofar as the mean distance between the lamellar overgrowths in the particular data that we employed for comparison of theory and experiment is a reasonable measure of the actual value of the mean characteristic crystallite length, the predictions concerning the axial ratio and the variation of characteristic length with undercooling appear to be approximately verified.

The theory allows the reduction in the dissolution temperature and heat of fusion resulting from cumulative volume strain to be calculated. The latter quantity can be utilized to estimate the expansion of the lattice in the crystallite caused by the volume strain in polyethylene fibrils formed in solution, and this is carried out and compared with experiment.

Under certain annealing conditions, the volume strain in the core fibrils can be relaxed, causing the reappearance of normal lattice spacings in the crystallites. The situations in which this is apt to occur are discussed, and the properties of the resultant fibrils outlined.

The presence of volume strain can affect the melting behavior of the fibrils. The treatment of Grubb and Keller is modified to account for this effect. The shape of the melting 
curves obtained by these authors from measurements of the contraction on heating of polyethylene fibrils can be reproduced by the revised theory, as well as by a theory where the volume strain is assumed to have been relaxed by annealing. This analysis leads to an independent determination of the characteristic length that is in fair accord with estimates based on the distance in fresh preparations between the lamellar overgrowths that decorate the core fibrils.

\section{Model}

We assume that in a suitable experiment a very long polymer chain in a solution is statistically elongated by the flow field, forming a convenient site (or more correctly, a set of sites) for nucleation (fig. 1A). This elongation will be most evident for the chains of greatest length. Specifically, we take these high molecular weight elongated chains as likely sites for the formation of a nucleus of the type shown in figure $1 \mathrm{~B}$, which we idealize as a square parallelepiped of diameter $a$ and length $l$, with the lateral surface free energy $\sigma$ and the bundle-end surface free energy $\sigma_{e}$ as shown. The value of $\sigma$ will be taken as similar to that of a normal lateral surface for a polymer crystal (circa $14 \mathrm{erg} / \mathrm{cm}^{2}$ for polyethylene), but the bundle-end surface free energy $\sigma_{e}$ after some growth must be expected to be certainly not less than and more probably somewhat greater than that for a strictly folded surface, the latter being about $93 \mathrm{erg} / \mathrm{cm}^{2}$ for polyethylene. The bulk of the crystallite is taken to be largely bundlelike or fringedmicellar in character, with numerous polymer chains emerging from the end surfaces and entering other crystallites (ciliary bridges). The interior of the crystallite (nucleus) will be partly of the extended-chain type, but with some chainend and chain fold defects.

Nuclei leading to crystallites of a similar type will form downstream on the same long central molecule (or on new and secondary polymer chains pendant downstream from the first nucleus or crystallite) giving rise after each nucleus grows to a connected set of long thin crystallites forming a very long core fibril (fig. 1C). These crystallites will nucleate on the pendant primary or secondary elongated chains at more or less random positions. It is important to see that new nuclei will also form between two nuclei that have already been established (see fig. 1A). Though the nuclei appear initially on the primary or secondary chains more or less at random, certain thermodynamic and kinetic considerations to be brought out subsequently mitigate in favor of something less than a totally random distribution of characteristic lengths under certain conditions. In particular, the average diameter and length of the crystallites will prove to be limited to a specified value even though a broad distribution about this mean value may still occur.

A special feature of the model that will be dealt with in detail later relates to the cumulative build up of surface stress at the bundle ends arising from increasingly strong repulsion of the ciliary bridges emanating from the end surfaces as the crystallite grows. This induces volume strain (at least while the tension resulting from the flow field is exerted on the fibril) and ultimately leads to a stable or metastable minimum in the free energy at a specified size of the crystallite.

We shall now begin to focus our attention on the formation of a specified nucleus on a primary or pendant secondary elongated chain.
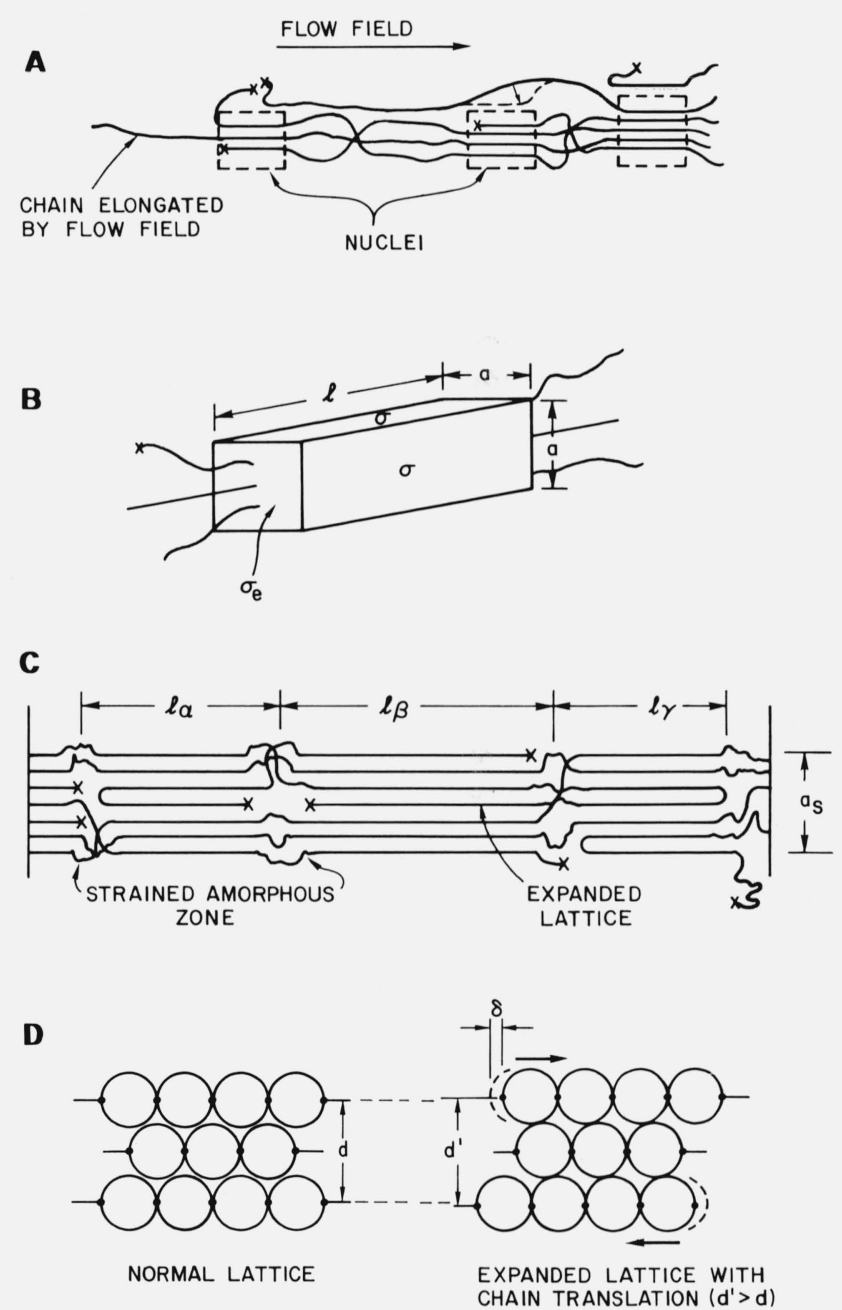

Figure 1. Formation and structure of core fibril.

(A) Bundlelike nuclei forming on polymer chains elongated by flow. (B) Geometry and surface free energies of nucleus or crystallite. (C) Schematic model of core fibril showing alternating crystalline and amorphous regions, the latter consisting largely of ciliary bridges. Note crossovers and other defects in the amorphous zones. The average value of the lengths $l_{\alpha}, l_{\beta}, l_{\gamma} \ldots$ is denoted $l_{s}$, and is termed the "characteristic length" in the text. Lamellar overgrowths not shown. Annealing may cause the cumulative strain to decay, allowing normal lattice spacings to appear (see text). (D) Lattice expansion effect caused by small lengthwise translations $\delta$ resulting from anisotropic tensions caused by differences in nature of ciliary bridges (e.g., crossovers) and defects in amorphous zone at either end of each molecule in core fibril crystallite. Small horizontal arrows represent direction of chain translation relative to reference (central) molecule (schematic). 


\section{Nucleation and Growth of the Core Fibril Crystallite with Cumulative Strain}

\subsection{Preliminary Considerations.}

It is convenient for future developments to consider first the conventional case of nucleation of an individual crystallite without cumulative strain. The free energy of formation of a crystallite of the shape shown in figure $1 \mathrm{~B}$ is

$$
\Delta \phi=2 a^{2} \sigma_{e}+4 a l \sigma-a^{2} l(\Delta f)
$$

where the free energy difference between the subcooled liquid and a very large and strain-free crystal is to a first approximation

$$
\Delta f=\frac{\left(\Delta h_{f}\right)\left(T_{d}^{\circ}-T\right)}{T_{d}^{\circ}}=\frac{\left(\Delta h_{f}\right)(\Delta T)}{T_{d}^{\circ}} .
$$

In these expressions, $\Delta \phi$ is in erg/crystallite, $a$ and $l$ in $\mathrm{cm}$, $\sigma$ and $\sigma_{e}$ in $\mathrm{erg} / \mathrm{cm}^{2}$ (millijoules $/ \mathrm{m}^{2}$ ), and the heat of dissolution (or fusion), $\Delta h_{f}$, in $\mathrm{erg} / \mathrm{cm}^{3}$ unless otherwise noted. The free energy of fusion, $\Delta f$, is also in erg $/ \mathrm{cm}^{3}$. The quantity $\Delta T$ is the undercooling $T_{d}^{\circ}-T$, and $T_{d}^{\circ}$ is the equilibrium dissolution temperature, analogous to the equilibrium melting temperature $T_{m}{ }^{\circ}$ for a bulk polymer. The value of $T_{d}^{\circ}$ is assumed to be corrected if necessary for the increase in this quantity that may result from the presence of the flow field. For the sake of simplicity, we have omitted a multiplying factor on the right-hand side of eq (2) of $f \cong 2 T$ / $\left(T_{d}^{\circ}+T\right)$ or $f=T / T_{d}^{\circ}$ that approximately corrects for the decrease in the heat of fusion with falling temperature.

By the customary procedure of setting

$$
\left(\frac{\partial \Delta \phi}{\partial l}\right)_{a}=0 ; \quad\left(\frac{\partial \Delta \phi}{\partial a}\right)_{l}=0
$$

it is readily found that the values of $l$ and $a$ at the saddle point in the $\Delta \phi-a-l$ surface (fig. 2A) described by eq (1) are

$$
a^{*}=4 \sigma /(\Delta f),
$$

and

$$
l^{*}=4 \sigma_{e} /(\Delta f)
$$

and that when these are inserted into eq (1) that the free energy at the saddle point is

$$
\Delta \phi^{*}=32 \sigma^{2} \sigma_{e} /(\Delta f)^{2} .
$$

An investigation of eq (1) shows that the value of $\Delta \phi$ falls after reaching $\Delta \phi^{*}$ and reaches a value of zero again at
A

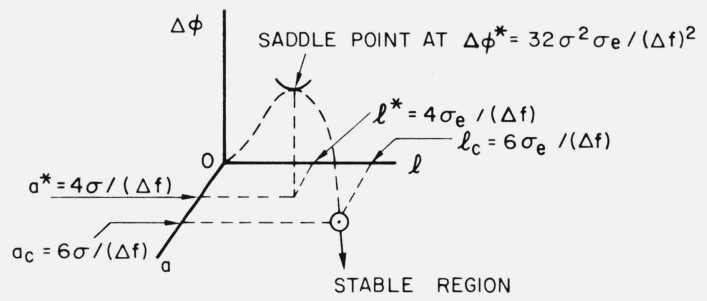

B

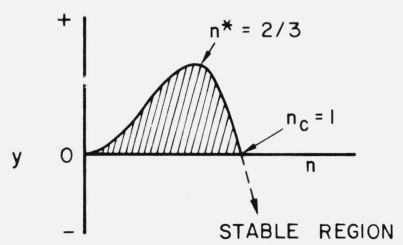

C

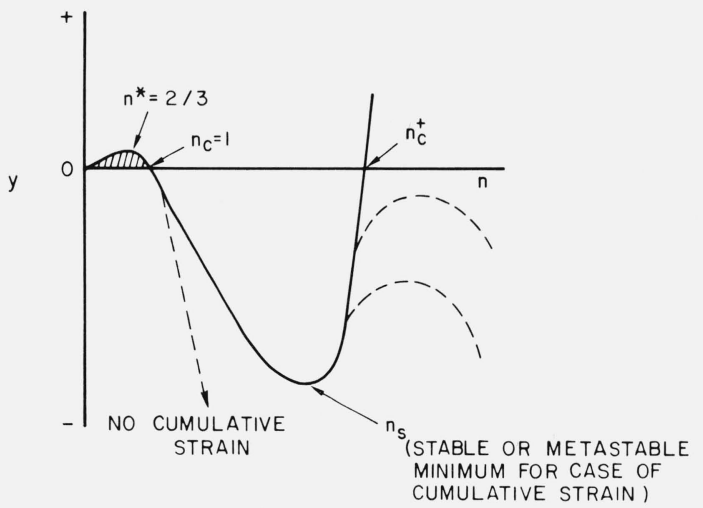

FigURE 2. Alternative representations of barrier system (schematic).

(A) Conventional $\Delta \phi-a-l$ plot of barrier system without cumulative strain. (B) $y-n$ space plot of same system. (C) $y-n$ space plot of system with cumulative strain showing stable or metastable minimum at $n_{s}$.

$$
a_{c}=6 \sigma /(\Delta f)
$$

and

$$
l_{c}=6 \sigma_{e} /(\Delta f) .
$$

This suggests a coordinate system where

$$
a=n \cdot \frac{6 \sigma}{(\Delta f)}
$$

and

$$
l=n \cdot \frac{6 \sigma_{e}}{(\Delta f)}
$$

These expressions allow $\Delta \phi$ as given in eq (1) to be expressed as

$$
\Delta \phi=\frac{216 \sigma^{2} \sigma_{e}}{(\Delta f)^{2}}\left\{n^{2}-n^{3}\right\}
$$


where we may define a reduced free energy of formation function as

$$
y=n^{2}-n^{3} .
$$

By simply taking $d y / d n=0$, it is found that $n^{*}=2 / 3$, corresponding to $\Delta \phi^{*}=32 \sigma^{2} \sigma_{e} /(\Delta f)^{2}$, and it is seen by inspection of eq (11) that $\Delta \phi=0$ when $n=1$, giving a crossover on the $n$ coordinate at $n_{c}=1$ (see fig. 2B). This convenient coordinate system will be used in the developments to follow.

Thus far, we have described a simple nucleation process that, once accomplished, leads to crystallite growth without limit in both the $a$ and $l$ dimensions in the stable region $(\Delta \phi$ or $y$ negative).

\subsection{The Form and Origin of Cumulative Strain.}

Crowding of the amorphous ciliary bridges at the bundle ends may be pictured in general as inducing equal biaxial stress on the ends of the crystallite (fig. 3C). (As will be discussed subsequently, the build-up of biaxial stress is also accompanied by a corresponding increase in inhomogeneous tensions originating in the amorphous zone at the bundle ends. These tensions tend to produce small lengthwise translations of the chains in the crystallite relative to one another; such translations have the important consequence of being able to distribute forces originating in the bundle ends long distances into the interior of the crystal.) Assuming an infinitesimal deformation of the crystallite resulting from the crowding phenomenon, Fong [14] proposed a continuum mechanical model relating the crowding-induced energy at the end surface to the dilatation-induced strain energy in an idealized transversely isotropic elastic crystal such that the end surface free energy takes the form

$$
\sigma_{e}=\sigma_{e o}+C\left(\frac{l^{\prime}}{l_{o}}\right)\left(\frac{V^{\prime}}{V_{o}}\right)^{2 / 3}+\ldots
$$

Here $\sigma_{e o}$ is the surface free energy in the absence of equal biaxial end surface stress, $C$ a material constant that we shall identify in a subsequent development as the end surface free energy associated with cumulative strain, $l_{0}$ and $V_{o}$ the original (unstrained) length and volume, respectively, $l^{\prime}, V^{\prime}$ the slightly larger length and volume that obtain upon the application of equal biaxial stress on the end surfaces, and the three dots denote higher order terms. The above expression applies to any material object of the type described, and does not yet explicitly contain the concept of cumulative strain.

It has been shown by Fong that classical formulations of strain energy functions of a deformable body such as eq (13) are not directly applicable to crystal growth problems since the latter involve increases of mass. By introducing a

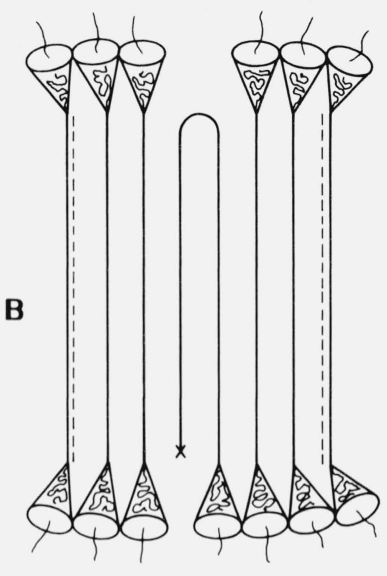

SMALL NUCLEUS

(NEGLIGIBLE CUMULATIVE)
STRAIN

LARGE CRYSTALLITE (WITH CUMULATIVE STRAIN )

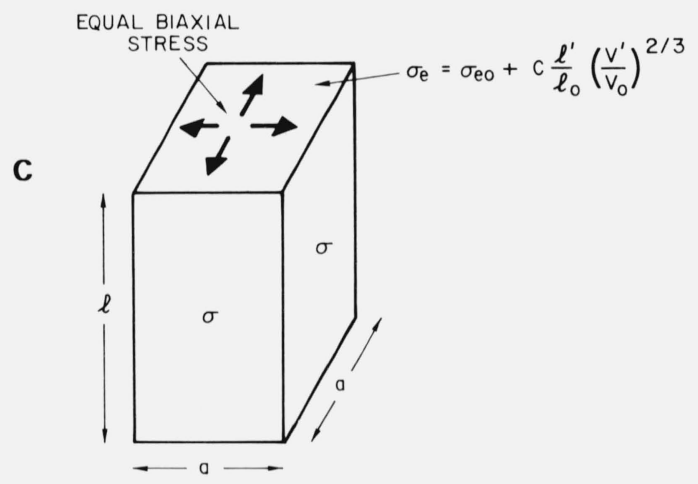

FigurE 3. Schematic representation of physical origin of cumulative stress.

In small nucleus depicted in (A), presence of sufficient number of chain ends, short cilia, or folds prevents repulsions leading to cumulative end-surface stress. Larger crystallite shown in (B) exhibits nonresolvable repulsions because of excessive number of emergent ciliary bridges and develops cumulative stress. Diagram (C) shows continuum mechanical model used to treat cumulative end surface stress and volume strain resulting from repulsion of cilia or ciliary bridges.

hypothetical intermediate state that is volume and shape preserving, but at the same time mass increasing, this author developed an argument using standard results of elasticity theory that modifies eq (13). This argument provides the required formula applicable to a crystallizing system, and at the same time provides the framework for introducing the cumulative strain approximation. This has a form similar to eq (13), and will be given subsequently.

It will prove to be important in the theory developed here that equal biaxial end stress can be associated with an expansion of the entire volume of the crystallite. It is relevant to note that Davis et al $[15,16]$ have clearly demonstrated by careful $x$-ray measurements that the presence of an end surface with its attendant surface free energy expands the lateral lattice spacings in the case of polyethylene. Specifically, their work shows that thin chain-folded single crystals 
exhibit larger lattice spacings (i.e., larger effective molecular cross-sectional areas) than do thick single crystals, and the same applies to chain-folded lamellae formed from the melt. The experimental constants describing the lattice expansion at a specified temperature as a function of lamellar thickness are nearly the same in these two cases [16]. A similar but considerably smaller effect occurs in the $n$-paraffins [16]. In all these instances, the expansion is essentially uniform in the sense that the increase in cross-sectional area is the same throughout the crystal, i.e., these systems behave as if they undergo equally distributed volume strain resulting from the presence of the end surfaces. It is also known that point defects such as $-\mathrm{CF}_{3}$ branches in poly(tetrafluoroethylene) have the general effect of expanding the entire lattice as has been shown by Bolz and Eby [17]. The latter point in particular shows that defects which are quite local in character can lead to relatively uniform expansion effects over considerable distances in systems consisting of chain molecules.

In the case of bundlelike core fibril crystallites, the postulated approximately uniform expansion effect (and a molecular explanation of the presence of the factor involving the length in the second term on the right-hand side of eq (13)) can be traced to inhomogeneities in the strained amorphous zone that cause small lengthwise translations of the chains in the crystallite relative to one another. Some of these inhomogeneities are of the type depicted in figure 1C. It is readily seen how such translations along the chain axis, even though much less than one chemical chain unit, can cause an expansion of the entire crystal, and not just a splaying near each end, if the chains are represented as linear sets of interlocking beads. Observe in figure $\mathrm{IC}$ that the ciliary bridges at either end of a given molecule in a crystallite are generally quite different-some go nearly straight through to a corresponding position in an adjacent crystallite, while others are longer and exhibit crossovers bordering on entanglements in character. This anisotropy is a direct result of the nature of the nucleation mechanism illustrated in figure 1A. Thus, as the end surface free energy builds up, a corresponding difference in tension builds up on the chains in the crystallite which tends to increasingly displace them lengthwise with respect to one another. (A torque can also lead to some chain translation.) Then if each chain is pictured as a set of beads, it is seen that a small lengthwise displacement of a chain of much less than one bead length relative to its neighbors will expand the mean distance between the chains throughout the entire length of the crystal. ${ }^{2}$ This is shown schematically in figure 1D. In the particular case of polyethylene, the direct cause of the expansion is the repulsion of the hydrogen atoms on one

\footnotetext{
${ }^{2}$ The explanation given above for the overall lattice expansion effect does not preclude the presence of some splaying near the crystallite ends resulting from the crowding of ciliary bridges. This effect would tend to be minimized by the fact that large separations require the loss of much of the heat of fusion in the affected regions.
}

chain with those on neighboring chains resulting from the small translation.

The foregoing discussion provides a rationale showing how forces at the bundle ends resulting from mutual repulsion of the ciliary bridges can lead to an additional component of the end surface free energy that depends, among other factors, on the length of the crystallite and which is at the same time associated with an expansion of the lattice i.e., volume strain. ${ }^{3}$ Comparatively large lattice expansion effects must be anticipated in bundlelike crystallites because of the considerable end surface stress in such systems. Mechanisms that may relax the volume strain will be noted subsequently.

It has long been suspected that a bundlelike or fringedmicellar crystal develops topological difficulties leading to energetically unfavorable conditions as it attempts to grow in size. Estimates concerning this question have previously centered on the magnitude of the end surface free energy, and it is now widely recognized that $\sigma_{e \text { (bundle) }}>\sigma_{e \text { (fold }}$ surface). The regularly folded surface for polyethylene exhibits a surface free energy close to $93 \mathrm{erg} / \mathrm{cm}^{2}$, and in the present work we estimate that $\sigma_{e \text { (bundle) }} \cong 120.5 \mathrm{erg} / \mathrm{cm}^{2}$. (The lattei figure refers only to the portion of $\sigma_{e \text { (bundle) }}$ not subject to cumulative strain, i.e., $\sigma_{e_{0}}$ in eq (13); a much higher figure to be quoted subsequently is obtained when results deriving from the second term in eq (13) are included.)

Some insight into the problem of the origin of the increased end-surface free energy is afforded by a consideration of the number of chains that can emanate from the end of a bundlelike crystal and still maintain configurations that at least roughly approximate random coil behavior. The number of polymer chains that can project from a flat surface and maintain liquid-like configurations is in fact quite limited. We define $f_{1}$ as the fraction of surface sites on the plane that can be occupied by long emergent chains with liquid-like character of the same cross-sectional area as each of the sites. DiMarzio [18] has commented that $f_{1}$ is probably less than one-half. Here however it is sufficient to refer to a calculation by Flory [19] who showed for one special case that

$$
f_{1} \cong 1 / 2
$$

In this case an emergent molecule was not allowed to take steps backward toward the plane of origin, so that the liquid

\footnotetext{
${ }^{3}$ Some intuitive understanding of the form of the second term on the right-hand side of eq (13) can be gained when it is seen that $C$ is in the units of surface free energy, and that $l$ must be a factor according to the arguments given concerning chain translation. For this second term to lead to a volume strain free energy effect when inserted into eq (1), it is readily shown that the multiplying factor in it must have a numerator with the dimensions of (length) ${ }^{2}$. The reasonable choices for this factor are $A^{\prime} J_{J}$ $A_{o}$, where $A^{\prime}$ and $A_{o}$ are the strained and unstrained total end areas, and $\left(V^{\prime} / V_{o}\right)^{2 / 3}$. In the case where one regards $l_{o}$ to change to $l^{\prime}$ with increasing tensions, one then has $C\left(l^{\prime} / l_{o}\right)\left(A^{\prime} / A_{o}\right)$ or $C\left(l^{\prime} / l_{o}\right)\left(V^{\prime} / V_{o}\right)^{2 / 3}$ as correct choices for the second factor. With even a very small lengthwise distension of the chains from $l_{o}$ to $l^{\prime}$ being allowed, and knowing that the end area must increase with chain translation, it may be surmised that the latter choice is correct. The continuum mechanical treatment of Fong [14] clearly decides in favor of $C\left(l^{\prime} / l_{o}\right)\left(V^{\prime} / V_{o}\right)^{2 / 3}$.
} 
state does not consist of random coils. The remaining fraction of sites $1-f_{1}$ on the surface are presumably occupied by chain folds or quite short cilia. Now if as in the model shown in figure $1 \mathrm{~A}$ and $\mathrm{IC}$, the fraction of chains emerging from an end surface is caused by multiple nucleation acts to exceed one-half, then the cilia (actually bridges between the nuclei or crystallites) would tend to repel one another, and excess surface stress would occur at the bundle ends as the crystallite grew.

In the case of fibril formation induced by flow, we are considering a situation where multiple nucleation essentially forces a mostly bundlelike mode of crystal growth on the system, so that a considerable fraction of the chains must emanate from the ends. Some folds may occur on the end surface, but turning a pendant molecule on the leeward end of a crystal back against the flow field to form a fold must be considered an improbable event except in the slow process of building up the nucleus itself to $n=2 / 3$ or $n=1$ (see fig. $2 \mathrm{~B})$. Further, the unattached portion of a molecule that is at points associated with one nucleus or crystallite on a flowaligned molecule will dangle as a secondary chain from this entity in a bundlelike mode, and attach downstream to one or more nuclei or crystallites in a similar manner (fig. 1A). Recall also our earlier remark that new nuclei formed at a later time between two previously established nuclei must necessarily exhibit a bundlelike character. The overall situation described clearly commits the system to a substantially bundlelike mode of crystallization where chain molecules perform traverses, i.e., bridges, from one crystallite or nucleus to another.

From the foregoing discussion, one must expect the majority of the molecules to partake of more than one nucleus or crystallite, such molecules passing through a highly strained "amorphous" zone between each crystallite. The overall result is that eq (14) is strongly violated, and cumulative stress must therefore be expected to develop at the bundle ends as the diameter and volume of each crystallite increases.

It is possible to indicate in physical terms, though still in a schematic way, what is occurring at the bundle ends. In the particular representation shown in figure 3 , the cilia (treated as one-half of a bridge between two nuclei or crystallites) are depicted as restricted to the volume of a cone, so that they are not random coils. The molecules in the cones are allowed to interpenetrate somewhat, but repulsions between them occur because the liquid density cannot be exceeded in the end surface layer. The result is that the effective cross-sectional area associated with each cone is larger than the cross-sectional area of the same molecule in the crystal proper. In figure $3 \mathrm{~A}$, the repulsion is reduced or absent because of the presence of folds and chain end defects, but in the larger crystal shown in figure $3 \mathrm{~B}$, the repulsion becomes more pronounced as the area of the crystal end increases. Thus, in a strictly bundlelike crystallite, or more correctly, a crystallite where the number of short cilia or folds in the end surface is insufficient to prevent violation of eq (14), cumulative stress must build up on that surface. It is seen that the difficulty in packing the cones for a large crystallite cannot be evaded by rounding the ends if it is remembered that ciliary bridges are involved. The longer ciliary bridges that would exist toward the edge would simply correspond to a larger set of cones in this region, and repulsions could not be avoided as the crystallite increased in diameter.

In the model, cumulative strain is not allowed to occur to a serious extent in the nucleus itself. We allow each nucleus to grow without strong cumulative strain up to the dimensions

$$
l_{c}=6 \sigma_{e o} /(\Delta f)
$$

and

$$
a_{c}=6 \sigma /(\Delta f)
$$

that is, to a volume $V_{c}=a_{c}^{2} l_{c}$. This corresponds to $n_{c}=1$ in figure 2 .

The physical concept underlying this scaling process is that the growth of the nucleus in the $\Delta \phi$ or $y$ positive region (shaded region in fig. $2 \mathrm{~B}$ and $2 \mathrm{C}$ ) is slow enough to allow some chain folds and short cilia or other defects to insert themselves into the bundle ends during the slow formation of the nucleus, allowing the longer cilia to be mostly free of cumulative repulsions (fig. 3A). When the volume exceeds $V_{c}$, growth becomes much more rapid and the crystallite is forced to accept the bundlelike end without recourse to processes that can mitigate the repulsions between the long cilia (fig. 3B). Thus, cumulative strain begins to manifest its major effect near $V_{c}$.

The results to be calculated are not particularly sensitive to the choice of the scaling point where cumulative strain becomes significant; we could have equally well chosen $a_{c}$ $=4 \sigma /(\Delta f)$ and $l_{c}=4 \sigma_{e o} /(\Delta f)$ as the physical dimensions corresponding to the situation where cumulative strain begins to be prominent with no basic change in physical interpretation and only trivial changes in the mathematical formulation.

We begin the actual treatment of cumulative strain by defining

$\epsilon=$ excess free energy of cilium or ciliary bridge (ergs/cilium)

and

$\nu=$ number of cilia or ciliary bridges

per unit area (number $/ \mathrm{cm}^{2}$ ) 
so that $\nu \epsilon$ is in the units of surface free energy. This defines the constant $C$ in eq (13) so we have

$$
\sigma_{e}=\sigma_{e o}+\nu \epsilon=\sigma_{e o}+\nu \epsilon_{c}\left(\frac{l^{\prime}}{l_{c}}\right)\left(\frac{V^{\prime}}{V_{c}}\right)^{2 / 3}
$$

where $\nu \epsilon_{c}$ is the value of the excess end surface free energy $\nu \epsilon$ at $n=1$, i.e., at $l=l_{c}, a=a_{c}$, and $V=V_{c}$. The assumption that $\nu \epsilon$ has the form $\nu \epsilon_{c}\left(l^{\prime} / l_{c}\right)\left(V^{\prime} / V_{c}\right)^{2 / 3}$ represents the first stage of what we denote the cumulative strain approximation, which is seen to be an explicit statement of the nature of the accumulation of free energy resulting from the repulsion of the ciliary bridges. An increase in $\nu \epsilon$ must be expected on physical grounds because of the crowding effect of the ciliary bridges as the end area and volume of the crystallite increases, as has been discussed. Equation (19) also exhibits the scaling hypothesis noted above, where we have set $l_{o}=l_{c}, V_{o}=V_{c}$. At this stage, eq (19) has not been justified for the case where the crystallite has grown. Recall also that $l^{\prime} / l_{c}$ and $V^{\prime} / V_{c}$ are only slightly different from unity.

We now wish to extend eq (19) to the case where the crystallite grows to a volume where the final volume $V$ is much greater than $V_{c}$. The second stage in the cumulative strain approximation consists essentially of assuming that at any given stage $i$ in the crystallite growth process the cilium surface free energy $\nu \epsilon$ is proportional to the length and the volume in the powers appropriate to eq (13) or (19). This can be given an explicit formulation.

Let us assume a stepwise growth process such that

$$
V_{c} \rightarrow V_{1} \rightarrow V_{2} \rightarrow V_{i} \cdots \rightarrow V_{j}
$$

where $V_{j}$ is now the final volume of the grown crystallite. A similar expression holds for the progression $l_{c} \rightarrow l_{1} \rightarrow l_{2} \cdots$ $l_{j}$. Then, according to our hypothesis, we may write

$$
\nu \epsilon\left(V_{1}, l_{1}\right)=\nu \epsilon\left(V_{c}, l_{c}\right)\left(\frac{l_{1}}{l_{c}}\right)\left(\frac{V_{1}}{V_{c}}\right)^{2 / 3}
$$

In going from $V_{1}$ to $V_{2}$, we get

$$
\nu \epsilon\left(V_{2}, l_{2}\right)=\nu \epsilon\left(V_{1}, l_{1}\right)\left(\frac{l_{2}}{l_{1}}\right)\left(\frac{V_{2}}{V_{1}}\right)^{2 / 3}
$$

Now by substituting $\nu \epsilon\left(V_{1}, l_{1}\right)$ from eq $(20 \mathrm{~b})$ into eq (20c), one finds

$$
\nu \epsilon\left(V_{2}, l_{2}\right)=\nu \epsilon\left(V_{c}, l_{c}\right)\left(\frac{l_{2}}{l_{c}}\right)\left(\frac{V_{2}}{V_{c}}\right)^{2 / 3}
$$

and in a similar way we obtain

$$
\nu \epsilon\left(V_{3}, l_{3}\right)=\nu \epsilon\left(V_{c}, l_{c}\right)\left(\frac{l_{3}}{l_{c}}\right)\left(\frac{V_{3}}{V_{c}}\right)^{2 / 3}
$$

leading to the general result that embodies the cumulative strain approximation

$$
\nu \epsilon\left(V_{j}, l_{j}\right)=\nu \epsilon\left(V_{c}, l_{c}\right)\left(\frac{l_{j}}{l_{c}}\right)\left(\frac{V_{j}}{V_{c}}\right)^{2 / 3} .
$$

Hence, recognizing $\nu \epsilon\left(V_{c}, l_{c}\right)$ is $\nu \epsilon_{c}$, we arrive at [14]

$$
\sigma_{e}=\sigma_{e o}+\nu \epsilon_{c}\left(\frac{l}{l_{c}}\right)\left(\frac{V}{V_{c}}\right)^{2 / 3}
$$

which is similar to eq (19), but with the additional justification through eqs (20) resulting from the cumulative strain approximation that it may be employed for a crystallite that has grown (with volume strain present) from $V_{c}$ to $V$ and $l_{c}$ to $l$, where the final volume $V$ (and length $l$ ) is now much larger than $V_{c}$.

Note that at any stage $i$ in the accretion process described by eqs $(20)$ that the ratio

$V_{i}^{\prime}$ (crystal with volume strain) $/ V_{i}($ unstrained crystal $)$

is always only slightly greater than unity; the appearance of values of $V / V_{c}$ many times greater than unity in eqs (20) and (21) does not imply an expansion in the lattice spacings of the crystallite resulting from cumulative strain equal to $V / V_{c}$ or $\left(V / V_{c}\right)^{2 / 3}$.

It is useful in understanding the nature of the cumulative strain approximation that is applicable to bundlelike crystals to indicate why it would not be employed in the case of $n$ paraffins and chain-folded crystals, the platelets of each of which grow without limit in the two " $a$ " dimensions, but not in the $l$ dimension. Even apart from the fact that $l$ is fixed in these cases, and the ratio $V_{\text {strain }}^{\prime} / V_{\text {no strain }}$ in the analog of eq (13) is replaced by a factor involving the ratio of the strained and unstrained areas, the procedure implied by eqs (20) cannot be applied. Equations (20) state directly that the effective end surface free energy $\nu \epsilon$ resulting from repulsion of cilia is a function of the length and volume of the crystal (see fig. 3), which is the essence of the cumulative strain approximation. This assumption cannot be made in cases where there are no physical grounds for deciding that the surface free energy depends on the dimensions of the system considered.

\subsection{The Free Energy Function With Cumulative Strain.}

Given the free energy of formation in ergs/crystallite as

$$
\Delta \phi=2 a^{2} \sigma_{e}+4 a l \sigma-a^{2} l(\Delta f)
$$

and inserting $\sigma_{e}$ from eq $(21)$, there is obtained 


$$
\begin{array}{r}
\Delta \phi=2 a^{2} \sigma_{e o}+4 a l \sigma-a^{2} l(\Delta f) \\
+2 a^{2}\left(\frac{l}{l_{c}}\right) \nu \epsilon_{c}\left(\frac{V}{V_{c}}\right)^{2 / 3}
\end{array}
$$

which becomes, on noting from eq (15) that $l_{c}=6 \sigma_{e o} /(\Delta f)$

$\Delta \phi=2 a^{2} \sigma_{e o}+4 a l \sigma-a^{2} l(\Delta f)$

$$
+a^{2} l(\Delta f) \alpha\left(V / V_{c}\right)^{2 / 3}
$$

where the volume strain parameter $\alpha$ is defined as

$$
\alpha \equiv \frac{\nu \epsilon_{c}}{3 \sigma_{e o}} .
$$

Observe in the foregoing formulation that the term $\nu \epsilon_{c}\left(l / l_{c}\right)\left(V / V_{c}\right)^{2 / 3}$ in $\sigma_{e}$ from eq (2l) has become a volume strain term in the free energy of formation (see last term in eqs (24) and (25)). The factor $\nu \epsilon_{c}$ may be regarded as the excess surface free energy at $n=1$ assignable to cumulative strain. Only the term $\sigma_{e 0}$ remains as an end surface free energy in the customary sense.

In order to obtain a simpler and more tractable expression, we introduce the numerical variables

$$
a=n_{a}[6 \sigma /(\Delta f)]
$$

and

$$
l=n_{l}\left[6 \sigma_{e o} /(\Delta f)\right]
$$

i.e., we have $n_{a}=a /[6 \sigma /(\Delta f)], n_{l}=l /\left[6 \sigma_{e o} /(\Delta f)\right], V=a^{2} l$ $=n_{a}^{2} n_{l}\left(216 \sigma^{2} \sigma_{e o}\right) /(\Delta f)^{3}$, and $V_{c}=216 \sigma^{2} \sigma_{e o} /(\Delta f)^{3}$. With these introduced into eq (25), we obtain

$$
\begin{aligned}
\Delta \phi & =\frac{216 \sigma^{2} \sigma_{e 0}}{(\Delta f)^{2}} \times \\
& \left\{\frac{1}{3} n_{a}^{2}+\frac{2}{3} n_{a} n_{l}-n_{a}^{2} n_{l}+\alpha\left(n_{a}^{2} n_{l}\right)^{2 / 3}\left(n_{a}^{2} n_{l}-1\right)\right\} .
\end{aligned}
$$

The term -1 has been subtracted from $n_{a}^{2} n_{l}$ in the last factor to account for the fact that cumulative strain occurs in the volume $V-V_{c}=\left[216 \sigma^{2} \sigma_{e o} /(\Delta f)^{3}\right]\left(n_{a}^{2} n_{l}-1\right)$. An alternative derivation of eq (28) that gives rise to this term in a natural way is given in the appendix (sec. 7). The term -1 has a negligible effect on the position of the stable minimum in the free energy in the calculations to follow; the advantage is that its use causes the reduced free energy function to be introduced below to be well-behaved near $n=0, n=2 / 3$, and $n_{c}=1$.

In the investigation of the properties of eq (28) to find maxima and minima in $\Delta \phi$ as a function of $n_{a}$ and $n_{l}$, we could proceed by calculating $\left(\partial \Delta \phi / \partial n_{a}\right)_{n_{l}}=0$ and $(\partial \Delta \phi /$ $\left.\partial n_{l}\right)_{n_{a}}=0$, but a simpler approach is warranted by the fact that the term involving $\alpha$ contains only factors involving powers of the volume. (In the notation in eq (28), $n_{a}^{2} n_{l}$ represents a reduced volume.) This means that the axial ratio of the crystallites is not affected by partial differentiation of the term involving $\alpha$, and we may therefore drop the subscripts $a$ and $l$ in eq (28). The results are in any case identical to that found using the partial derivatives of $\Delta \phi$ with respect to $n_{a}$ and $n_{l}$ noted above.

Dropping the subscripts in eq (28) and collecting terms of like powers of $n$, we arrive at the working equation

$$
\Delta \phi=\frac{216 \sigma^{2} \sigma_{e o}}{(\Delta f)^{2}}\left\{n^{2}-n^{3}+\alpha n^{2}\left(n^{3}-1\right)\right\} .
$$

Note that when $\alpha=0$, corresponding to no cumulative strain, eq (11) is recovered.

It is now possible to calculate the salient properties of the model. These include: (1) the position of the minimum $n_{s}$ and the crossover at $n_{c}{ }^{+}$in $y-n$ space (see fig. 2C); (2) the mean axial ratio of the crystallites; (3) the mean diameter and mean characteristic length of the individual crystallites making up the core fibril; (4) the depression of the dissolution temperature and heat of fusion; (5) an estimate of the lattice expansion incurred by the cumulative strain and (6) melting and annealing behavior. In dealing with the latter, we shall also address the problem of the distribution in crystallite lengths.

\section{Properties of the Model}

\subsection{Stable Minimum and Other Characteristics of the Reduced Free Energy Function.}

From eq (29), it is seen that the reduced free energy function with cumulative volume strain is

$$
y=n^{2}-n^{3}+\alpha n^{2}\left(n^{3}-1\right) .
$$

We first examine eq (30) for crossovers at $y=0$ with emphasis on those other than which occur at $n_{c}=0$. The reason for investigating $y(n, \alpha)$ for crossings of the $y$ coordinate is clear from figure $2 \mathrm{C}$; a crossover at large $n$ implies that a minimum exists between $n_{c}=1$ and the larger value of $n$, which we call $n_{c}{ }^{+}$. Though fluctuations will occur, the system average will never actually attain the crossover at large $n$, but will seek instead the minimum in $\Delta \phi$ that exists between $n=1$ and $n_{c}{ }^{+}$.

The crossover at $n_{c}{ }^{+}$is found by setting $y=0$, corresponding to $\Delta \phi=0$, taking two roots of $n=0$, and another at $n$ $=1$, after which it is found that

$$
\alpha n^{2}+\alpha n+\alpha-1=0 .
$$


The relevant root is

$$
n_{c}^{+}=\frac{1}{2} \sqrt{\frac{4-3 \alpha}{\alpha}}-\frac{1}{2} \cong \frac{1}{\alpha^{1 / 2}}-\frac{1}{2} .
$$

The approximation shown is quite acceptable since $\alpha$ for polyethylene is close to 0.01 (see later).

We now investigate eq (30) for the existence of the stable minimum. By calculating $d y / d n$, setting the result equal to zero, and taking one root at $n=0$, it is seen that

$$
y^{\prime}=5 \alpha n^{3}-3 n+2-2 \alpha=0 .
$$

Surmising a root very close to $n-2 / 3=0$, one finds to a sufficient approximation that

$$
5 \alpha n^{2}+(10 / 3) \alpha n-3+20 \alpha / 9=0 .
$$

The root $n^{*}=2 / 3$ represents a maximum in $y(n, \alpha)$ at $\Delta \phi^{*}$ (fig. 2C). The stable minimum is represented by the following root of eq (34)

$$
n_{\text {stable }}=n_{s}=\frac{1}{3} \sqrt{\frac{5.4}{\alpha}-3}-\frac{1}{3} \cong \frac{0.7746}{\alpha^{1 / 2}}-\frac{1}{3} .
$$

For values of $\alpha$ in the vicinity of 0.01 , i.e., values of $n_{s}$ in the vicinity of roughly 7 , the approximate expression for $n_{s}$ is correct to within 0.6 percent.

A plot of $y$ versus $n$ for $\alpha=0.015$ and $\alpha=0.0118$ is given in figure 4 using eq (30). It is seen that the plot for $\alpha$ $=0.0118$ gives a stable minimum at $n_{s}=6.77$. The minimum occurs at $y_{s}=-99.2$. The crossover $n_{c}{ }^{+}$occurs at 8.70 for $\alpha=0.0118$. We have emphasized the case giving $n_{s}=6.77$, since it will emerge later that this is the experimental value of $n_{s}$ for polyethylene fibrils. Note in figure 4 that the larger value of $\alpha$ gives a less stable miminum at a smaller value of $n_{s}$. Calculations for other values of $\alpha$ show that this trend is general. The practical upper bound for $\alpha$ is about $0.1-$ this gives $n_{s} \sim 2$.

A plot of the function given by eq (30) in the region of $n$ $=0$ and $n_{c}=1$ is shown in the inset of figure 4 for some $\alpha$ values. It is seen that $y$ is a well-behaved function in this region, and exhibits a maximum corresponding to a nucleation barrier very close to $n^{*}=2 / 3$. The barrier height is however slightly lower for $\alpha>0$ than for $\alpha=0$.

The overall picture deduced from eq (30) is that an embryo forms and slowly grows by fluctuations in the $y$ or $\Delta \phi$ positive region to the barrier maximum at $n^{*}=2 / 3$. After surmounting the nucleation barrier, the relatively more rapid growth process begins. Rapid accretion of new molecules commences at $n_{c} \cong 1$, where the free energy turns negative. Rapid growth then proceeds, and the crystallite strives toward the minimum at $n_{s}$ (figs. $2 \mathrm{C}$ and 4 ).

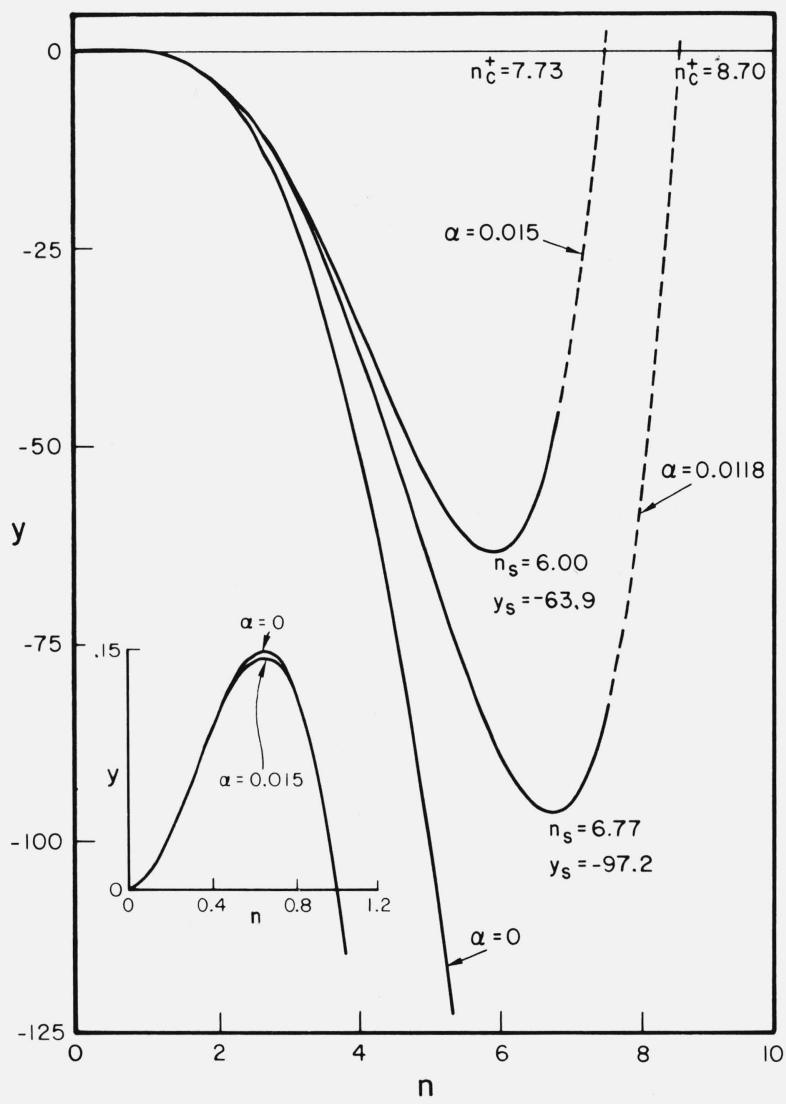

FIGURE 4. Plot of reduced free energy function y against $n$ for various values of strain parameter $\alpha$ according to eq (30).

Inset shows detail of behavior of $y$ between $n=0$ and $n=1$.

\subsection{Stable Crystallite Dimensions and Dependence on Undercooling.}

Recalling that $l=n\left[6 \sigma_{e 0} /(\Delta f)\right]$ and $a=n[6 \sigma /(\Delta f)]$, we immediately find that the stable value of the mean characteristic length is

$$
\begin{aligned}
l_{\text {stable }}=l_{s}=n_{s}\left(\frac{6 \sigma_{e o}}{\Delta f}\right) & \\
& \cong\left(\frac{0.7746}{\alpha^{1 / 2}}-\frac{1}{3}\right) \frac{6 \sigma_{e 0} T_{d}^{\circ}}{\left(\Delta h_{f}\right)(\Delta T)} .
\end{aligned}
$$

The mean value of the core fibril diameter is

$$
\begin{aligned}
a_{\text {stable }}=a_{s}=n_{s}\left(\frac{6 \sigma}{\Delta f}\right) & \\
= & \left(\frac{0.7746}{\alpha^{1 / 2}}-\frac{1}{3}\right) \frac{6 \sigma T_{d}^{\circ}}{\left(\Delta h_{f}\right)(\Delta T)} .
\end{aligned}
$$

According to eq (36), we may write

$$
l_{s}=C_{1} /(\Delta T)
$$


where

$$
C_{1}=\left(\frac{0.7746}{\alpha^{1 / 2}}-\frac{1}{3}\right) \frac{6 \sigma_{e 0} T_{d}^{\circ}}{\left(\Delta h_{f}\right)}
$$

and from eq (37), we get

$$
a_{s}=C_{2} /(\Delta T)
$$

where

$$
C_{2}=\left(\frac{0.7746}{\alpha^{1 / 2}}-\frac{1}{3}\right) \frac{6 \sigma T_{d}^{\circ}}{\Delta h_{f}}
$$

We observe from eqs (36) and (37) that the mean axial ratio $\gamma$ of each crystallite is given by

$$
\gamma=\frac{l_{s}}{a_{s}}=\frac{\sigma_{e o}}{\sigma} .
$$

The expressions noted above represent some of the key predictions of the theory. In qualitative terms, the treatment implies the following. At high undercoolings, $(\Delta T$ large $)$, the basic core fibrils will be quite thin, and at lower undercoolings, they will have a larger diameter. Further, because $\sigma_{e o}$ $>\sigma$, and the relation $\sigma_{e o} / \sigma=l_{s} / a_{s}$, the mean characteristic length $l_{s}$ will always be considerably larger than the diameter of the fibril; for polyethylene, one must expect $\gamma \geq \sim 6.5$ $\left(\sigma_{e o} \geq \sigma_{e}=93 \mathrm{erg} / \mathrm{cm}^{2}\right.$ and $\left.\sigma=14 \mathrm{erg} / \mathrm{cm}^{2}\right)$. The characteristic length (which in special cases we will interpret as the mean distance between the lamellar platelets or "kabobs" in a properly prepared specimen) will increase with decreasing undercooling in a manner closely parallel to the increase of the diameter of the fibril. Further, we must expect both $l_{s}$ and $a_{s}$ to be many times larger than the primary nucleus, mainly because of the factor $n_{s} \sim 0.7746 /$ $\alpha^{1 / 2}$ in egs (36-41). The theory predicts that the mean value of the axial ratio should be approximately independent of the undercooling (or driving force $\Delta f$ ).

Experiments where fibrils are formed are invariably carried out under conditions where a flow field is present, and it is well known that a large flow field can substantially increase the driving force $(\Delta f)$ for crystallization [6]. In practical but still qualitative terms, this may be taken as equivalent to an increase in the dissolution temperature. Thus, at a constant temperature, the effective undercooling $\Delta T$ can be increased by increasing the flow field (e.g., increasing take-up speed or rotor velocity). In such an example, increasing the take-up speed at a constant temperature should at least at first tend to cause a decrease in diameter and characteristic length of the fibrils. The real situation is however quite complicated, especially for the stirrer method, and it is not a simple matter to quantitatively determine the value of the dissolution temperature and hence the undercooling for this method. Complex hydrodynamic effects can complicate matters further at high rotor velocities [6]. The properties of fibrils formed in highly inhomogeneous and strong flow fields could easily lie outside the range of validity of the theory presented here. The present theoretical developments are probably best tested where a low and constant flow field is used, and where the undercooling is changed by varying the crystallization temperature ${ }^{4}$. In this case the trend of $\Delta T$ is definitely known, and the actual value of $\Delta T$ can be estimated at least within broad limits. In such experiments, the prediction is that an increase of crystallization temperature will lower the undercooling, and lead to an increase in fibril diameter and characteristic length.

It is important to qualify any attempted identification of the mean characteristic length $l_{s}$ of the crystallites with the mean distance between the lamellar platelets that decorate the core fibril. There are actually two simple hypotheses that would lead to at least an approximate correspondence of these two quantities. One which has already been cited is that of George and Tucker, who assumed that cilia associated with the discontinuities in the core fibril nucleate the lamellar overgrowths. Another hypothesis that allows $l_{s}$ to be similar to the mean lamellar overgrowth distance is that the overgrowth explicitly avoids the amorphous regions, and preferentially nucleates on the core fibril proper, mostly on the basis of one overgrowth per crystallite. Again, such overgrowths might tend to be epitaxial in character. In either case, the success of identifying $l_{s}$ with the mean distance between lamellar overgrowths depends on the avoidance of additional overgrowths as much as possible, since such extra platelets would lead to an erroneously low estimate of $l_{s}$. Depending on which of the above hypotheses is thought to apply, the distribution functions describing the spacing of the overgrowths and the amorphous regions may be nearly the same or quite different, and still exhibit essentially the same mean value.

In comparing theory and experiment, we would accordingly prefer to deal with interlamellar platelet distance data where the formation of the core fibrils and overgrowths was quite rapid, and where fast quenching was then immediately employed in such a manner as to freeze the solvent into the solid state. Such a procedure should minimize any excessive number of platelet overgrowths, and at the same time curtail the type of rearrangements of the lamellar overgrowth that have been described by Pennings, Lageveen, and Vries [13]. The experiments of George and Tucker meet these requirements. It will be shown in section 5 that the hypothesis that

\footnotetext{
${ }^{4}$ The filament take-up method, where the fibril is grown continuously, has reasonable prospects for allowing analysis of the effects of variations of intensity of flow field at constant temperature (see paper by Zwijnenburg et al [20]). At the present writing it is still difficult to estimate the value of $\Delta f$ in such experiments, but they would appear to involve a more uniform flow field than for example the stirrer method at high rotor velocities. The techniques advanced by Andersen and Carr [21] may prove useful in estimating $\Delta f$ in such systems.
} 
the average distance between the lamellar overgrowths is associated with the mean distance between the amorphous zones in the core fibril in suitable preparations is a reasonable one.

Perhaps the most important observation concerning the theory at this stage is that it provides a natural explanation for the limited diameter exhibited by the basic core filament even in a supersaturated solution. The fibril does not grow radially for the lack of nutrient polymer, but actually exhibits a stable (or metastable) diameter because of cumulative strain originating ultimately from topological and energetic considerations at the bundle ends.

Another point is that the theory leads in a natural way to the type of structure shown in figure $1 \mathrm{C}$ where a significant fraction of the long polymer chains go through many different crystallites. This is generally consistent with the enormous tensile strength exhibited by single fibrils. The treatment allows some defects (e.g. folds) to concentrate at the crystallite ends. Chain end defects can occur everywhere, though the concentration is apt to be higher in or near the amorphous regions, or just external to these regions (fig. 1C). The molecular arrangement shown in figure $1 \mathrm{C}$ bears a strong general resemblance to that proposed by Grubb and Keller [11]. As pointed out earlier, the crossovers and entanglements in the amorphous zone are a natural result of the nucleation and growth process depicted in figure 1 .

We observe that eq (42) is consistent with the crystal shape that obtains by minimizing the total surface free energy of a square parallelipiped crystal of any fixed volume $V_{k}$ that has surface free energies $\sigma$ and $\sigma^{\prime}{ }_{e}$. This arises as follows. Taking

$$
\Delta \phi_{\text {crystal }}=2 a^{2} \sigma_{e}^{\prime}+4 a l \sigma-a^{2} l(\Delta f)
$$

and employing an arbitrary fixed volume $V_{k}=a^{2} l$, one obtains

$$
\Delta \phi_{\text {crystal }}=2 a^{2} \sigma^{\prime}{ }_{e}+4 V_{k} \sigma / a-V_{k}(\Delta f) .
$$

By setting $\left(\mathrm{d} \Delta \phi_{\text {crystal }} / \mathrm{d} a\right)=0$ for fixed $V_{k^{\prime}}$ one gets $a \sigma^{\prime}{ }_{e}=$ $V_{k} \sigma / a^{2}$, which, with $a^{2}=V_{k} / l$, leads directly to

$$
\frac{l}{a}=\frac{\sigma_{e}^{\prime}}{\sigma} .
$$

Here $l$ and $a$, unlike $l_{s}$ and $a_{s}$ in eq (42), are unrestricted dimensions. Equation (45) is a simple embodiment of Wulff's theorem, but it is clear that this theorem cannot alone explain the limited size, or the temperature-dependence of that size, of the basic units of the polymer fibril.

Conversion of the results showing a stable minimum in the $y-n$ plot to the more conventional $\Delta \phi-a-l$ coordinate system is easily carried out and the general result of performing this transformation is shown schematically in figure 5. The minimum in the $y-n$ plot at $n_{s}$ becomes a deep trench elongated in the $l$ direction in the $\Delta \phi-a-l$ representation where the minimum has the coordinates $\Delta \phi_{s}$, $a_{s}, l_{s}$. The value of $\Delta \phi$ at the minimum is $\left\{216 \sigma^{2} \sigma_{e o} /(\Delta f)^{2}\right\}$ - $y_{s}$ where it will be recalled that $y_{s}$ is negative. Steep walls rise everywhere from the bottom of the trench. The $\Delta \phi-a-l$ representation exhibits the conventional saddle point at $\Delta \phi^{*}, a^{*}, l^{*}$.

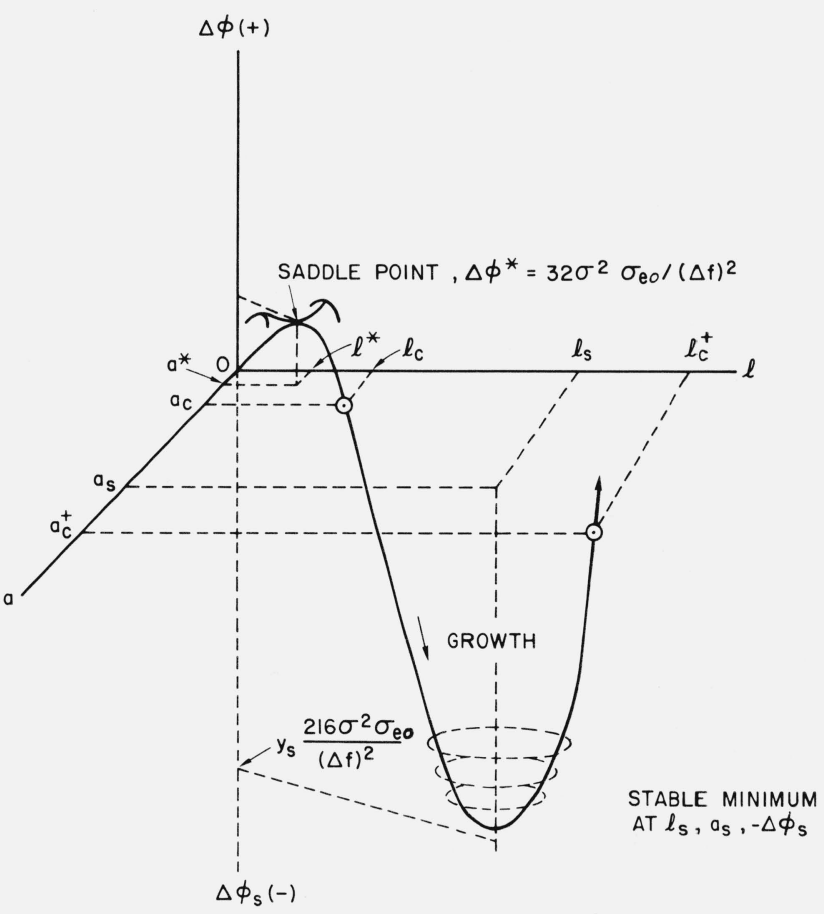

Figure 5. Plot of $\Delta \phi-a-l$ space for models with cumulative strain (schematic).

Finally, we concern ourselves with the range of validity of eq (25) or (28). Because of the terms involving $\left(V / V_{c}\right)$ (or $\left.n_{a}^{2} n_{l}\right)$ in these expressions, the free energy does not go without limit to more negative values as the volume increases, but instead exhibits a minimum in the negative free energy regime at a certain value of $n$. It is believed that this minimum is real in an ordinary time frame and exists for the physical reasons stated. Nonetheless, it is necessary to qualify the use of the term "stable" that we have often used in connection with it. The term "metastable" is probably more precise. The permanence of the minimum depends on whether or not in sufficient time the cumulative strain can be dissipated. Mechanisms for doing so can in fact be envisioned. If such mechanisms exist, they may provide routes to a more stable state where the volume strain is reduced and one or more of the dimensions of the crystal is larger (see dotted line in fig. 2C). In such a case, the minimum is a metastable one. If such routes are not accessible to the 
system in a specified time frame, the minimum is "stable," and the continued rise in free energy at $n>n_{s}$ up to $n_{c}{ }^{+}$is real. For convenience, we have mostly used the term "stable" to describe the minimum, but the provisos noted here should be understood in connection with its use.

An entirely different sort of limitation should be noted. Under a fixed set of external conditions, there will be a practical upper limit to the temperature where core fibrils can be formed in a reasonable time because the nucleation rate will in effect fall off rather abruptly to an inconsiderable value. Also, at sufficiently low temperatures the nucleation rate will become very high, and probably lead to a lower temperature bound below which fibrils cannot properly form; at these low temperatures, competition with ordinary homogeneous nucleation could also occur and mask or prevent fibril formation.

\subsection{The Distribution of Crystallite Lengths.}

Prior to discussing certain aspects of the melting behavior of the assembly of crystallites that comprise the core fibril, it is convenient to introduce the concept that some variation will occur in the diameter and especially in the length of the crystallites. We concentrate on the topic of the distribution of lengths, since certain details of the melting behavior depend on this function.

It was noted in the discussion of the basic model that the nuclei were assumed to be first deposited more or less at random on the primary elongated molecule, or on secondary chains dangling from the first or any subsequent nucleus or crystallite. If some process did not intervene, and this distribution were frozen in, it would tend to give a quite broad distribution of lengths for the matured crystallites. Also one would expect a very large variation of the average value of $l$ with temperature if it were solely controlled by nucleation events. The nuclei would be widely spaced at low undercoolings and much more densely spaced at high undercooling. But we have just shown in eqs (36) and (38) that $l$ will actually tend toward a stable or metastable value of $l_{s}$, which implies that the system of crystallites (while quite possibly still having a broad distribution as a result of a memory of the original nucleation acts) should exhibit a mean length that is related to $l_{s}$. This implies that, whatever the distribution, the mean value is bounded. At this stage of the development of the theory, we have not calculated the natural distribution of $l$ about $l_{s}$ resulting from fluctuations, but surmise that it can be considerably more narrow than that laid down in the initial nucleation process, especially at low undercoolings. Because of the elongated shape in the $l$ direction of the trench near the stable minimum in the $\Delta \phi$ $-a-l$ representation, fluctuations in $l$ will probably be considerably larger than those in $a$. In any event, the original distribution of lengths resulting from nucleation on primary and secondary pendant chains is certainly very broad, but there exists a natural driving force of thermodynamic origin toward a more truncated distribution. More important, we must expect the mean value of the distribution of lengths (characteristic length) to be reasonably close to $l_{s}$.

Grubb and Keller [11] have suggested the distribution function

$$
w(l)=\frac{l}{(\bar{l})^{2}} e^{-l l} \bar{l}
$$

where $w(l)$ is the mass fraction of crystallities of length $l$ that occurs in the distribution, and $\bar{l}$ is a length parameter that corresponds to the peak value of the distribution. This distribution is very broad, and rather asymmetric, and may be taken as a simple and practical representation of the shape of the initial distribution resulting from more or less random nucleation on the primary elongated chain. Pennings has observed that the distribution of lamellar platelet distances in certain samples follows the logarithmic-normal distribution, and remarks that there is some theoretical justification for its choice [6]. The distribution function suggested by Grubb and Keller is quite similar in general shape (i.e., it is broad and has a similar type of asymmetry) and its use is simplified by the presence of only one parameter, $\bar{l}$.

The average value of $l$ in the mass distribution employed by Grubb and Keller is

$$
\langle l\rangle=\int_{o}^{\infty} l w(l) d l=2 \bar{l}
$$

and the average value of $l^{2}$ is

$$
\left\langle l^{2}\right\rangle=\int_{0}^{\infty} l^{2} w(l) d l=6 \bar{l}^{2} .
$$

The coefficient of variation for this function is always

$$
C V \equiv \frac{100 \sqrt{ }\left\langle l^{2}\right\rangle-\langle l\rangle^{2}}{\langle l\rangle}=\frac{100 \sqrt{2}}{2}=70.7 \%
$$

Grubb and Keller were able to reproduce the shape of melting curves for fibrillar polyethylene with a broad $l$ distribution with a particular choice of $\bar{l}$ and other parameters [11].

\subsection{Melting Behavior and Some Aspects of Annealing.}

A fibril consisting of crystallites of the type depicted in figure $1 \mathrm{~B}$, but with no cumulative strain, has the dissolution 
temperature

$$
T_{d}=T_{d}^{\circ}\left\{1-\frac{2 \sigma_{e}}{\left(\Delta h_{f}\right) l}-\frac{4 \sigma}{\left(\Delta h_{f}\right) a}\right\}
$$

as can be found be setting $\Delta \phi=0$ in eq (1). The dissolution temperature for the case where cumulative strain exists can be obtained by setting $\Delta \phi=0$ in eq (25) and noting that $\left(V / V_{c}\right)^{2 / 3}=n^{2}=n_{s}^{2}$. The result is

$$
\begin{aligned}
T_{d}=T_{d}^{\circ}\left\{1-\frac{2 \sigma_{e o}}{\left(\Delta h_{f}\right) l_{s}\left(1-\alpha n_{s}{ }^{2}\right)}\right. & \\
& \left.-\frac{4 \sigma}{\left(\Delta h_{f}\right) a_{s}\left(1-\alpha n_{s}{ }^{2}\right)}\right\} .
\end{aligned}
$$

The quantity $\alpha n_{s}^{2}$ is 0.541 for polyethylene, and $\sigma_{e o}$ is about $120.5 \mathrm{erg} / \mathrm{cm}^{2}$; estimates of $\sigma_{e}$ in eq (48) range from $\sim 1445$ $\mathrm{erg} / \mathrm{cm}^{2}$ to $\sim 550 \mathrm{erg} / \mathrm{cm}^{2}$ for this polymer, depending on the degree of annealing (see later).

Because of the condition $\sigma_{e o} / \sigma=l_{s} / a_{s}$ that obtains for the crystallites with volume strain, $T_{d}$ as given in eq (49) may be written in the alternative and equivalent forms

$$
\begin{aligned}
T_{d}=T_{d}^{\circ}\left\{1-\frac{6 \sigma_{e o}}{\left(\Delta h_{f}\right) l_{s}\left(1-\alpha n_{s}^{2}\right)}\right\} \\
=T_{d}^{\circ}\left\{1-\frac{6 \sigma}{\left(\Delta h_{f}\right) a_{s}\left(1-\alpha n_{s}^{2}\right)}\right\} .
\end{aligned}
$$

In writing eqs (49) and (50), it has been assumed that the volume strain in the crystallites is not relaxed prior to dissolution or melting. In all of the expressions quoted here, the melting point of a dried specimen is given by replacing $T_{d}$ with the observed melting point $T_{m}$, and $T_{d}^{\circ}$ with $T_{m}^{\circ}$.

It is worth pointing out that if the strained amorphous region were destroyed in some manner and converted to short chain ends, the volume strain would be relaxed. Also $\sigma_{e o}$ would fall, and become comparable to $\sigma$. In this case the dissolution temperature would closely approach

$$
\begin{aligned}
T_{d}=T_{d}^{\circ}\left\{1-\frac{4 \sigma}{\left(\Delta h_{f}\right)}\left(\frac{1}{a_{s}}+\frac{1}{2 l_{s}}\right)\right\} & \\
& \cong T_{d}^{\circ}\left\{1-\frac{4 \sigma}{\left(\Delta h_{f}\right) a_{s}}\right\} .
\end{aligned}
$$

Such a set of circumstances should thus cause a considerable increase in the dissolution temperature (or melting point) compared with that of the original untreated fibrils. Equation (51) offers an opportunity to obtain an experimental estimate of $\sigma$ from melting point measurements on suitably treated specimens of different diameter. A plot of $T_{d}$ or $T_{m}$ versus $1 / a$ has a slope close to $4 T_{d, m}{ }^{\circ} \sigma / \Delta h_{f}$, and an intercept close to $T_{d, m}$. With $\Delta h_{f}$ being known, then $\sigma$ is known to a good approximation. This method of estimating $\sigma$ is altogether analogous to the $T_{d}$ or $T_{m}$ versus $1 / l$ plot method of obtaining $\sigma_{e(\text { fold })}$ for chain-folded single crystals [22, 23].
The foregoing expressions apply to melting or dissolution temperatures that have been freed of the effects of superheating. Also, they do not explicitly take into account any annealing effects that may occur that may lead to an increase in crystallite size and perfection. Further, we have ignored the melting of the chain-folded platelets, but the melting of these objects can usually be distinguished from that of the core fibril.

At this juncture, it is essential to note the probable effects of annealing, since certain phenomena that are associated with this process can alter the melting behavior and the presence of volume strain. The most probable result of the first stages of annealing is some relaxation of the repulsion of the ciliary bridges that cause the high end surface free energy and volume strain. In polyethylene, such behavior could be caused by the advent of lengthwise translational motions in the crystal associated with the $\alpha$-relaxation mechanism, wherein a molecule shifts one $\mathrm{C}-\mathrm{C}$ repeat distance and rotates $180^{\circ}$ [24], which becomes active just above $\sim 100^{\circ} \mathrm{C}$. An interesting situation can arise from this which is best illustrated by the example of polyethylene fibrils: on annealing, the volume strain may dissipate, leaving only a large end surface free energy with the amorphous zone essentially intact. An explanatory discussion follows.

Consider first the free energy of formation of an unstrained crystal, i.e., one in which the lattice is not expanded and where the strain is concentrated in the bundle ends. This is represented by eq (1) and is herewith labelled $\Delta \phi_{u}=$

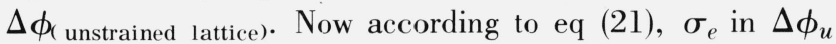
may be written as $\sigma_{e 0}+\left(\nu \epsilon_{c}\right) n_{s}^{3}$ using $l / l_{c}=n_{s}$ and $(V /$ $\left.V_{c}\right)^{2 / 3}=n_{s}^{2}$. The quantity $\sigma_{e}$ is $120.5+4.27(6.77)^{3}=$ $1445 \mathrm{erg} / \mathrm{cm}^{2}$ for polyethylene (see later). Now for the corresponding crystallite of the same dimensions exhibiting volume strain we use eq (25), and denote the free energy of formation in $\Delta \phi_{s}=\Delta \phi_{\text {(strained lattice) }}$ for the purposes of this discussion. The quantity $\alpha\left(V / V_{c}\right)^{2 / 3}=\alpha n_{s}^{2}$ in eq (25) is 0.541 and $\sigma_{e o}$ is $120.5 \mathrm{erg} / \mathrm{cm}^{2}$ for polyethylene. It is easily shown either numerically or by using the relations $\alpha \equiv$ $\left(\nu \epsilon_{c} / 3 \sigma_{e o}\right)$ and $l=n_{s}\left[6 \sigma_{e o} /(\Delta f)\right]$ that $\Delta \phi_{u}=\Delta \phi_{s}$ when $\sigma_{e}$ in $\Delta \phi_{u}$ is $\sigma_{e o}+\left(\nu \epsilon_{c}\right) n_{s}^{3}$. Thus, a polyethylene crystallite exhibiting volume strain with $\sigma_{e 0}=120.5 \mathrm{erg} / \mathrm{cm}^{2}$ has exactly the same free energy of formation as an unstrained crystal with no lattice expansion with an end surface free energy of $\sigma_{e}=1445 \mathrm{erg} / \mathrm{cm}^{2}$. Now if some annealing takes place and $\sigma_{e}$ falls, then the unstrained crystallite will exhibit the lower free energy. Thus, a certain degree of annealing, which may occur concurrent with formation of the fibril itself, especially in preparations of long duration or at high growth temperatures, can readily lead to a collapse of the expanded lattice in fibrillar specimens. We also observe that even a small degree of mechanical tension colinear with the core fibril axis will tend to stabilize the form with volume strain, if prior to the application of tension $\sigma_{e 0}$ and $\sigma_{e}$ in eqs 
(21) and (1), respectively, are such that $\Delta \phi_{s}=\Delta \phi_{u}$. (The stabilization of the state with volume strain is apt to occur during the formation process because of the tension on the fibril caused by the flow field.) The tension has the physical effect of increasing the forces that translate the chains and increase the volume. The above observations imply that the crystallites exhibiting volume strain may be ephemeral, with the greatest chance of their being found residing in fibrils formed in brief periods at low temperatures, and probably in a state of tension.

Further insight on annealing can be gained by extending the discussion to certain simple aspects of melting behavior. If we substitute $\sigma_{e}=\sigma_{e 0}+\left(\nu \epsilon_{c}\right) n_{s}^{3} \cong 1445 \mathrm{erg} / \mathrm{cm}^{2}$ in eq (48), which represents a crystallite without volume strain, a considerably lower melting point is obtained than is found with eq (50) with $\sigma_{e o}=120.5 \mathrm{erg} / \mathrm{cm}^{2}$ and $\alpha n_{s}{ }^{2}=0.541$, the latter applying to a crystallite with volume strain. Thus, in the absence of annealing effects, the strained crystal would be found in a melting run. However, it is easily shown that when $\sigma_{e}$ in eq (50) falls (presumably as a result of annealing) to a value near $550 \mathrm{erg} / \mathrm{cm}^{2}$ that the melting point calculated for the unstrained state using eq (48) is higher than that found with eq (50) for the strained state. Accordingly, annealing can cause a collapse of the expanded lattice, but with a large end surface free energy of $\sigma_{e} \sim 550 \mathrm{erg} / \mathrm{cm}^{2}$ still being present that gives the same melting point as the original strained crystallite. (Calculations for $\sigma=14.4 \mathrm{erg} /$ $\mathrm{cm}^{2}, a_{s}=300 \AA P, l_{s}=2400 \AA$.) In such a case, the unstrained crystal phenomena would be found in melting experiments. We infer from the foregoing discussion that $\sigma_{e}$ $\sim 550 \mathrm{erg} / \mathrm{cm}^{2}$ is a reasonable estimate for the end surface free energy of a polyethylene fibril crystallite that has just transformed from the volume-strained to the volume-unstrained state in a melting run. A surface free energy of this magnitude without volume strain can have significant effects on melting behavior, as will be shown subsequently.

A discussion will be given later concerning the possible ultimate effects of prolonged annealing near the melting point.

We now address the subject of the shape of the melting curves of the core fibril, especially as these are affected by the distribution in $l$, and the presence of volume strain. Our treatment parallels that given by Grubb and Keller and utilizes their distribution function as displayed as eq (46); the principal difference is that cumulative strain is included in the present case. By employing the concepts given by Grubb and Keller, but using the free energy function given by eq (25) rather than eq (1) (which except for unimportant geometrical considerations is the same as that used by them) one finds

$$
\chi\left(l_{o}\right)=\int_{l_{o}}^{\infty} w(l) d l=\left(1+\frac{l_{o}}{\bar{l}}\right) e^{-l_{o} / \bar{l}}
$$

as given by Grubb and Keller, but $l_{0}$ takes on the revised form:

$$
l_{o}=\frac{2 \sigma_{e o}}{\left[\left(T_{m}^{\circ}-T_{o}\right)\left(\Delta h_{f}\right)\left(1-\alpha n_{s}^{2}\right) / T_{m}{ }^{\circ}\right]-4 \sigma / a_{s}} .
$$

This is the Grubb-Keller formulation with volume strain from the present theory included. In these expressions, $\chi\left(l_{0}\right)$ is the fraction unmelted, and $T_{0}$ is the melting point of a crystallite of length $l_{o}$. The treatment ignores the effect of any distribution in the fibril diameter. The above expressions lead to a quite broad melting curve for reasonable choices of $\bar{l}_{e}$. Recall that according to the present formulation $\bar{l}$ is restricted by eq (47a) to a value in the vicinity of one-half of the mean characteristic length. Since $\sigma_{e o}, \sigma, T_{m}{ }^{\circ}$ and $\Delta h_{f}$ are known, one test of the theory is accomplished by reproducing a melting curve using only $\bar{l}$ as a fitting parameter, and determining if that $\bar{l}$ corresponds through eq (47a) to a reasonable value of the mean characteristic length.

In view of the foregoing discussion concerning the effects of annealing, we reproduce here for further use the expression for $l_{0}$ for the case where the volume strain has relaxed and where the cumulative stress remains concentrated at the bundle ends:

$$
\begin{gathered}
l_{o}=\frac{2 \sigma_{e}}{\left[\left(T_{m}{ }^{\circ}-T_{o}\right) \Delta h_{f} / T_{m}{ }^{\circ}\right]-4 \sigma / a_{s}} \\
\quad \text { (volume strain absent) }
\end{gathered}
$$

This is essentially the formula of Grubb and Keller for the parallelepiped geometry used here. ${ }^{5}$ This expression will prove useful where it is suspected that relaxation of the volume strain has taken place. Such relaxation could take place either in the process of a high temperature preparation, or prolonged storage at a lower temperature, or during the melting run itself. In the latter case, we expect $\sigma_{e} \sim 550$ $\mathrm{erg} / \mathrm{cm}^{2}$ as noted previously.

\subsection{Depression of the Heat of Fusion Resulting from Volume Strain.}

In a calculation to follow concerning the amount of lattice expansion in a core fibril crystallite caused by the presence of cumulative strain, it is necessary to have an estimate of the fractional depression of the heat of fusion attributable to the volume strain. We assume that the volume strain is principally enthalpic in character, and that the decrease in enthalpy is proportional to that part of the decrease in melting temperature caused by the strain. In this approximation, the fractional decrease in the heat of fusion is

\footnotetext{
${ }^{5}$ A minor difference is caused by the fact that Grubb and Keller employ the relation $\Delta f=\left[\left(\Delta h_{f}\right)(\Delta T) / T_{m}^{\circ}\right]\left(T / T_{m}{ }^{\circ}\right)$ in deriving their analog of eq (53b). See remarks concerning $(\Delta f)$ in section 3.1.
} 


$$
\begin{aligned}
f_{h} & \equiv \frac{\Delta H_{f}-\Delta H_{f \text { (strain) }}}{\Delta H_{f}} \\
& =\frac{\Delta T_{\text {(strain) }}-\Delta T_{\text {(no strain) }}}{T_{d}^{\circ}} \\
& =\frac{6 \sigma_{e o}}{\left(\Delta h_{f}\right) l_{s}\left(1-\alpha{n_{s}}^{2}\right)}-\frac{6 \sigma_{e o}}{\left(\Delta h_{f}\right) l_{s}} \\
& =\frac{6 \sigma_{e o}}{\left(\Delta h_{f}\right) l_{s}}\left(\frac{\alpha n_{s}{ }^{2}}{1-\alpha n_{s}^{2}}\right) .
\end{aligned}
$$

Here $\Delta H_{f}$ is the heat of fusion in $\mathrm{Jg}^{-1}$ in the absence of volume strain, and $\Delta H_{f(\text { strain }}$ the corresponding quantity with volume strain. The symbol $\left(\Delta h_{f}\right)$ is in $\mathrm{erg} / \mathrm{cm}^{3}$ and refers to the case of no strain. Because of the relation given in eq (42), the factor $6 \sigma_{e o} /\left(\Delta h_{f}\right) l_{s}$ can be replaced by $6 \sigma /$ $\left(\Delta h_{f}\right) a_{s}$. The value of $f_{h}$ is typically about 0.01 to 0.04 for normal values of the parameters, the larger depression being associated with the thinner fibrils. Notice that $f_{h}=0$ for $\alpha$ $=0$, as must be the case.

\subsection{Estimate of the Lattice Expansion Resulting from Volume Strain in the Core Fibril.}

Because a connection exists between the reduction in the heat of fusion caused by surface effects and the concomitant expansion of the lattice, it is possible to develop a scheme to predict the lattice expansion in a fibril in terms of the strain parameter $\alpha$. For reasons that will become apparent, this part of the development is aimed specifically at the case of polyethylene, but is believed to have more general validity.

From their work on the $\mathrm{x}$-ray spacings of lamellar bulk polyethylene samples and single crystals, Davis, Eby, and Colson [15] have shown that the thermodynamic properties depend on the fold period $l_{2}$. In particular, they have given the heat of fusion in $\mathrm{Jg}^{-1}$ of polyethylene as

$$
\Delta H_{f(\text { strain })}=\Delta H_{f}-K_{1} / l_{2}
$$

where $l_{2}$ is in $\AA, \Delta H_{f}$ is the heat of fusion in $J^{-1}$ of a sample of infinite lamellar thickness (i.e., with no strain) at the temperature under consideration, and $\Delta H_{f(\text { strain })}$ the heat of fusion of a specimen with the finite lamellar thickness $l_{2}$ that exhibits volume strain. (The volume strain in a chain-folded single crystal or in folded melt-crystallized polymer is of course non-cumulative.) Values of $K_{1}$, which they report in units of $\mathrm{Jg}^{-1} \AA$, at temperatures of interest can be obtained directly from their figure 9 , and $\Delta H_{f}$ is known. At $23{ }^{\circ} \mathrm{C}, K_{1}$ is $160 \mathrm{Jg}^{-1} \AA$. The value of $K_{1}$ is a bulk property of the crystalline polyethylene substance itself, and is at any given temperature independent of whether the orthorhombic crystal involved is paraffinic, chain-folded, or bundlelike. In deriving the basis for eq (55), Davis and coworkers assumed that the product (absolute temperature ${ }^{-c o e f f i c i e n t}$ of expansion·isothermal bulk modulus) was much greater than the pressure; this is equivalent to the assumption $\Delta E \cong \Delta H$, which is probably only a fair approximation in the present application.

In a subsequent paper, Davis, Weeks, Martin, and Eby [16] demonstrated experimentally that the cross-sectional area $A_{o}$ occupied by each chain is given with high accuracy by

$$
A_{o(\text { strain })}=A_{o}+K_{2} / l_{2} \text {. }
$$

Here $A_{o}$ is the cross-sectional area of the chain in an undisturbed lattice at a specified temperature in $\AA^{2}$ for polyethylene, and $A_{o \text { (strain) }}$ the same quantity for a sample with a finite lamellae of thickness $l_{2}$ that exhibits an expanded lattice resulting from end surface stress. ${ }^{6}$ The value of $K_{2}$ in $\AA^{3}$ has been given by these authors for meltcrystallized polymer, solution crystallized polymer, and $n$ paraffins, and highly precise values of $A_{o}$ are available from the same source [16]. The magnitude of $K_{2}$ for melt-crystallized polymer and solution crystallized polymer centers around $15.7 \AA^{3}$ at $23{ }^{\circ} \mathrm{C}$, but $K_{2}$ for the $n$-paraffins is much lower. The value of $K_{2}$ is not known from direct experiment for a bundlelike crystal, but a reasonable estimate can be made beginning with the aforementioned data on chainfolded systems. It is understood that $K_{2}$ in the formulae to follow refers to a bundlelike crystal.

By eliminating $l_{2}$ from eqs (55) and (56) and recalling that $\left\{\Delta H_{f}-\Delta H_{f(\text { strain })}\right\} / \Delta H_{f}=f_{h}$, it is found that

$$
A_{o(\text { strain })}=A_{o}+\frac{K_{2}}{K_{1}}\left(\Delta H_{f}\right) f_{h}
$$

which with $\Delta H_{f} /\left(\Delta h_{f}\right)=10^{-7} / \rho_{\mathrm{c}}$, where $\rho_{\mathrm{c}}$ is the crystal density in $\mathrm{g} / \mathrm{cm}^{3}$, gives

$$
\begin{aligned}
A_{o(\text { strain })} & =A_{o}+\frac{K_{2}}{K_{1}} \cdot \frac{10^{-7}}{\rho_{c}} \cdot \frac{6 \sigma_{e o}}{l_{s}}\left(\frac{\alpha n_{s}{ }^{2}}{1-\alpha n_{s}^{2}}\right) \\
& =A_{o}+\frac{K_{2}}{K_{1}} \cdot \frac{10^{-7}}{\rho_{c}} \cdot \frac{6 \sigma}{a_{s}}\left(\frac{\alpha n_{s}{ }^{2}}{1-\alpha n_{s}{ }^{2}}\right) .
\end{aligned}
$$

With the above formula, it will prove possible to obtain a rough estimate of the increase in volume (mostly crosssectional area) incurred by cumulative volume strain.

Both $K_{1}$ and $K_{2}$ depend on the temperature, and by referring to the original references, it is possible to calculate the lattice expansion at various temperatures.

${ }^{6}$ One direct cause for the lattice expansion in chain-folded systems is probably the torque exerted by the folds which then leads to small chain translations. Another probable cause is non-cumulative repulsions of the folds that cause similar translations. This expansion is naturally associated with a corresponding reduction in the heat of fusion. 
The extrapolation from the small lattice expansions actually observed in single crystals and melt crystallized polyethylene to the considerably larger one found in fibrillar material is a long one. Also, the assumption $\Delta E \sim \Delta H$ has been employed in estimating $K_{1}$. Another difficulty is that $K_{2}$ is not known for fibrillar specimens, though it is certainly somewhat larger than the $K_{2}$ value quoted by Davis et al for single crystals or melt-crystallized polyethylene. The value of $K_{2}$ can be corrected in an empirical manner so that it more accurately represents a bundlelike crystallite by multiplying it by the ratio $\sigma_{e o} / \sigma_{e \text { (fold) }}$. Given the approximations noted, it would probably be fortuitous if eqs (57) quantitatively predicted results that were within better than a factor of two of the experimental data.

Two qualitative features of eqs (57) are worth noting. First, the theory suggests that the larger expansions (i.e., effective cross-sectional areas) will occur in the thinner fibrils. The second and more essential point is that a rather marked expansion of the lattice is expected; this may amount to several percent or more for thin fibrils. The expansion is large enough to consider the possibility that the volume strain induced by the crowding of the ciliary bridges at the bundle ends may in some cases suffice to induce a phase transition to a more open lattice type of higher symmetry. ${ }^{7}$

Together with the previously given variation of crystallite dimensions with undercooling, the prediction of considerably expanded lattice spacings in the body of the core fibril represents a major point of the present approach that can be tested experimentally. The observation of a considerably expanded lattice in a thin fibril would support the general approach and model given here. Recall, however, that annealing mechanisms may cause a disappearance of the lattice expansion effect while still leaving the other principal features of the fibril intact, as was noted in section 4.4. (We would judge from the temperature and duration of the preparations commonly used in forming polyethylene fibrils from solution that the expanded lattice effect would not ordinarily appear in the specimens after the flow field was removed because of annealing phenomena.) We also remark that if the strained amorphous regions in the core fibril were somehow selectively destroyed, say by chemical attack, the strain in the crystal proper would be relieved, and relatively normal lattice parameters should result. (See also remarks on melting behavior in connection with eq (53).)

\footnotetext{
${ }^{7}$ As noted in Section 4.4, the application of mechanical tension on the core fibril may stabilize the expanded lattice. It follows that the application of such tension may in special cases either return a collapsed lattice to the expanded and strained state, or induce a transition to a new lattice of higher symmetry. We observe further that the longer crystallities in a fibril clamped at a low temperature to constant length will be subjected to strong tension as the shorter crystallites melt out on warming, this tension being a result of the contractile force due to randomization in the melted regions. Fibrils treated in this manner may exhibit the expanded lattice or even a phase transition to a higher symmetry form. (See discussion of contractility in sec. 5.5). The occurrence of such phenomena in clamped fibers would provide strong support for the treatment proposed in this paper.
}

\section{Comparison of Theory with Experiment}

\subsection{General}

Data are not available to test all aspects of the theory, but a number of the most important predictions can be compared with experiment. In particular, it is possible to deal in a quantitative manner with the variation of the diameter and mean characteristic length with undercooling, the predicted invariance of the mean axial ratio with temperature, and the expansion of the fibril lattice resulting from the presence of cumulative strain. The general effect of a change of the intensity of the flow field at constant temperature can also be dealt with qualitatively. We have already made the point that the presently available evidence is consistent with the fact that the fibril diameter has a definite limiting value indicative of the presence of a stable (or metastable) minimum in the free energy function as predicted by the theory. Melting phenomena, including the shape of the melting curves are also discussed, mainly with the objective of obtaining an independent estimate of the characteristic length. Experiments that indicate that the amorphous zones can be mostly removed by prolonged annealing at elevated temperatures are noted.

\subsection{Dependence of Fibril Diameter and Characteristic Length on Undercooling.}

George and Tucker [12] have measured the mean diameter $a_{s}$ of the fibrils that were produced by mild shear of a $3^{1 / 2}$ percent solution of polyethylene in $n$-octadecane at $84{ }^{\circ} \mathrm{C}$ and $101{ }^{\circ} \mathrm{C}$. They also measured the mean distance between the lamellar platelets at these two temperatures, which we take to approximate the characteristic length $l_{s}$ (table 1 ). The crystallization technique employed involved shearing a thin film of the polymer solution between two mica strips on which carbon had been evaporated that were held at the crystallization temperature, followed by quick quenching to room temperature. The shearing step was completed in less than about $1 \mathrm{~s}$, and the quench effected in a few seconds. The solid $n$-octadecane was then selectively removed at room temperature by use of a suitable solvent, leaving the polyethylene fibrils for examination.

We chose these particular experiments for consideration for a number of reasons. Among these was that identical crystallization conditions were employed at the two temperatures cited, and the coefficient of variation of the measurements were given at both temperatures. Also, the technique that was employed evidently minimized adventitious epitaxial decoration of the core filament. (The assumptions under which the mean distance between the lamellar overgrowths can approximate the mean length of the crystallites have been noted previously.) We also note that rather mild shear conditions were employed in the experiments, with the result 
that we did not judge it necessary to apply a large correction for the increase in $T_{d}^{\circ}$ that might have been caused by the shear. The general effect on the results of making an upward correction to $T_{d}^{\circ}$ is given.

We would propose that the formation of fibrils under the conditions of mild shear described above may be related to the fact that some portion of the longest molecules present will have some of their segments physically adsorbed on the glass surfaces. In such a case, the flow field would be very effective in statistically elongating those sections of the adsorbed molecules that were not so attached to these surfaces.

The test of the theory is begun by determining if eqs (38) and (40) can be used to fit the data at the two undercoolings used by George and Tucker with reasonable values of the parameters. Using the value of $T_{d}^{\circ}$ given in table 1 , which is that of Huseby and Bair [22], it is found with eq (40) that

$$
a_{s}(\mathrm{~cm})=8.41 \times 10^{-5} /(\Delta T)=C_{2} /(\Delta T)
$$

closely reproduces the experimental data (see table 1). By using the standard values [23] of $\sigma$ and $\Delta h_{f}$ listed in table 1 in conjunction with eqs (37) and (41), it is found that $n_{s}=$ 6.77 and $\alpha=0.0118$.

Attention is now directed to the $l_{s}$ data. A fair fit of the experimental data in table 1 can be obtained with eq (38) as

$$
l_{s}(\mathrm{~cm})=7.04 \times 10^{-4} /(\Delta T)=C_{1} /(\Delta T) .
$$

The datum $l_{s}=2514 \AA$ for $T=101{ }^{\circ} \mathrm{C}$ was weighted more heavily in calculating $C_{1}$ in eq (59) because of its smaller $\mathrm{CV}$ (see details in table 1), and the greater possibility of additional overgrowths in the sample formed at the lower crystallization temperature. With $C_{1}$ and $\alpha$ (or $n_{s}$ ) known, it is a simple matter to calculate that $\sigma_{e o}$ is $120.5 \mathrm{erg} / \mathrm{cm}^{2}$ using eq (41).

The fit of $a_{s}$ and $l_{s}$ shown in table 1 is sufficiently good to warrant the belief that each varies approximately as the reciprocal of the undercooling. The largest error occurs for $l_{s}$ at the highest undercooling (1550 ̊ calc., 1293 obs.). This low value of $l_{s}$ may be a result of some extra overgrowths.

The value $\sigma_{e o}=120.5 \mathrm{erg} / \mathrm{cm}^{2}$ may be regarded as reasonable for the cumulative strain-free component of the end surface free energy; it is somewhat larger than the fold surface free energy $\sigma_{e(\text { fold })} \cong 93 \mathrm{erg} / \mathrm{cm}^{2}$.

The theory predicts that the mean value of the axial ratio of the crystallites comprising the core fibril will be constant at different undercoolings, and that the axial ratio is $\sigma_{e o} / \sigma$. Using eq (42), we have for the mean axial ratio

$$
\gamma=\frac{\sigma_{e o}}{\sigma}=\frac{120.5}{14.4}=8.37
$$

where $\sigma$ and $\sigma_{e o}$ are the surface free energies that lead to the correct absolute values of $a_{s}$ and $l_{s}$ at the two undercoolings using eqs (34) and (37). The result shown in eq (60a) is in reasonable agreement with the direct length and diameter measurements of $\gamma$ by George and Tucker, who determined

$$
\gamma=\left(\frac{l_{s}}{a_{s}}\right)_{a v .}=\begin{gathered}
7.5 \text { at } 84{ }^{\circ} \mathrm{C} \\
8.7 \text { at } 101{ }^{\circ} \mathrm{C}
\end{gathered}
$$

The value of $\gamma$ is approximately independent of temperature

\begin{tabular}{|c|c|c|c|c|c|}
\hline \multicolumn{6}{|c|}{ A. General } \\
\hline \multicolumn{2}{|c|}{ Quantity } & & \multicolumn{2}{|c|}{ Value } & \\
\hline \multicolumn{2}{|c|}{$\begin{array}{l}\text { Heat of fusion, } \Delta h_{f} \\
\text { Lateral surface free energy, } \sigma \\
\text { Dissolution temperature of polyethylene in } n \text {-octadec- } \\
\quad \text { ane, } T_{d}^{\circ}\end{array}$} & & \multicolumn{2}{|c|}{$\begin{array}{l}2.80 \times 10^{9} \mathrm{erg} / \mathrm{cm}^{3} \\
14.4 \mathrm{erg} / \mathrm{cm}^{2} \\
129.4{ }^{\circ} \mathrm{C} \text { or } 402.6 \mathrm{~K}\end{array}$} & \\
\hline \multicolumn{6}{|c|}{ B. Fibril Dimensions at Various Undercoolings } \\
\hline \multirow{2}{*}{$\begin{array}{c}\text { Crystallization } \\
\text { temperature, } T\left({ }^{\circ} \mathrm{C}\right)\end{array}$} & \multirow{2}{*}{$\begin{array}{l}\text { Undercooling, } \\
\qquad \Delta T\left({ }^{\circ} \mathrm{C}\right)\end{array}$} & \multicolumn{2}{|c|}{$\begin{array}{c}\text { Fibril Diameter, } \\
a_{s}(\AA)\end{array}$} & \multicolumn{2}{|c|}{$\begin{array}{l}\text { Characteristic } \\
\text { length, } l_{s}(\AA)\end{array}$} \\
\hline & & Exptl. [12] & Theor. & Exptl. [12] & Theor. \\
\hline $\begin{array}{r}101 \\
84\end{array}$ & $\begin{array}{l}28.4 \\
45.4\end{array}$ & $\begin{array}{l}303(29 \% \mathrm{CV}) \\
181(51 \% \mathrm{CV})\end{array}$ & $\begin{array}{l}296 \\
185\end{array}$ & $\begin{array}{l}2514(17 \% \mathrm{CV})^{a} \\
1293(58 \% \mathrm{CV})\end{array}$ & $\begin{array}{l}2478 \\
1550\end{array}$ \\
\hline \multicolumn{6}{|c|}{ C. Derived Results } \\
\hline \multicolumn{2}{|c|}{$\begin{array}{l}\text { (1) } n_{s}=6.77 \\
\text { (2) } \alpha=0.0118 \\
\text { (3) } \sigma_{e o}=120.5 \mathrm{erg} / \mathrm{cm}^{2}\end{array}$} & & \multicolumn{2}{|c|}{$\begin{array}{l}\text { (4) } \nu \epsilon_{c}=4.27 \mathrm{erg} / \mathrm{cm}^{2} \\
\text { (5) } \alpha n_{s}^{2}=0.541 \\
\text { (6) }\left(\nu \epsilon_{c}\right) n_{s}^{3}=1325 \mathrm{erg} / \mathrm{cm}^{2}\end{array}$} & \\
\hline
\end{tabular}
as predicted. It may be concluded that the relation $\gamma=\sigma_{e o} /$ $\sigma=l_{s} / a_{s}$, eq (42), is generally consistent with the observa-

TABLE 1. Input data and results for dimensions of polyethylene core fibrils as a function of crystallization temperature and undercooling.

${ }^{a}$ The $l$ value of $2514 \AA$ was weighted $(58 / 17)^{2}=11.3$ times more than $l=1293 \AA$ in fitting $l{ }_{s}$ as a function of $1 / \Delta T$. 
tions, and the expectations of the theory concerning the magnitude of $\sigma$ and $\sigma_{e o}$.

It is of interest to indicate the sensitivity of the results to the estimate used for $T_{d}^{\circ}$. If $5{ }^{\circ} \mathrm{C}$ is arbitrarily added to the value of $T_{d}^{\circ}$ in table 1 , the following results are obtained: $C_{1}$ $=8.245 \times 10^{-4} \mathrm{~cm} \cdot \mathrm{deg}, C_{2}=9.62 \times 10^{-5} \mathrm{~cm} \cdot \mathrm{deg}, \gamma=$ 8.57, and $\sigma_{e o}=123.4 \mathrm{erg} / \mathrm{cm}^{2}$. These estimates of $\gamma$ and $\sigma_{e o}$ are still physically reasonable, but the fit of the data points for $a_{s}$ and $l_{s}$ is not as good. The theory would remain tenable from the standpoint of general physical interpretation even if $10^{\circ} \mathrm{C}$ were arbitrarily added to the $T_{d}^{\circ}$ value given in table 1 .

At this stage, the following conclusions are apparent. The variation of both the mean fibril diameter and mean characteristic length are in reasonable accord with theory, which states that they should vary as the reciprocal of the undercooling, and bear a constant ratio between themselves as they do so. This ratio is given by $\sigma_{e 0} / \sigma$, which is large because of the large natural difference between the bundleend and lateral surface free energies. The cumulative strainfree component of the bundle-end surface free energy is of the expected magnitude.

\subsection{Dependence of Fibril Dimensions on Flow Field Intensity at Constant Growth Temperature.}

Zwijnenburg, van Hutten, Pennings and Chanzy [20] have measured by various techniques the diameter and the mean distance between the lamellar overgrowths for polyethylene fibrils formed continuously from a 0.5 percent solution in p-xylene at a temperature of $103{ }^{\circ} \mathrm{C}$, but at two different filament take-up rates. The results are shown in table 2 .

The fibril diameter data are reasonably consistent with one another and have an unambiguous meaning. The length data denoted TEM in table 2 refers to the mean distance between the lamellar overgrowths. Our identification of these length data with the characteristic length $l_{s}$ involves the same assumption as was used by George and Tucker in analyzing their data. The lengths denoted SAXS in table 2 were obtained from a very intense small-angle reflection which the authors identified with the distance between the overgrowths. Some of this intense reflection may have been generated by the amorphous regions that presumably exist in the core fibril beneath the lamellar overgrowths.

The theoretical prediction is that the larger take-up rate should induce the larger value of $\Delta f$, and through eqs (36) and (37), produce the thinner and shorter core crystallites. This is borne out by the data in table 2 .

The data in table 2 imply an axial ratio for each take-up speed of $\gamma \sim 6$, in rough agreement with the findings of George and Tucker as well as with the value $\gamma \geqq \sim 6.5$ estimated on theoretical basis. The results noted in this section are in general consistent with the conclusions drawn in section 5.2.

Although it has no direct bearing on comparison of the present theory with experiment, mention should be made of the fact that observations by dark field electron microscopy and the broadening of the 002 electron diffraction spots for polyethylene fibrils lead to the postulate of a dimension in the chain direction that is much smaller than $l_{s}$. For instance, Zwijnenburg and coworkers [20] find for the same specimens listed in table 2 the dimension $250 \AA$ for a takeup speed of $2 \mathrm{~cm} / \mathrm{min}$, and 340 to $350 \AA$ for a take-up speed of $8 \mathrm{~cm} / \mathrm{min}$. They conclude that the elementary core fibril possesses orthorhombic "blocks" of this length. They indicate that this effect may be a result of crystal flaws, or possibly twist disclinations. There is no evidence suggesting that there is an actual hiatus in the crystal structure between these blocks, such as exists at either end of the crystallite of length $l_{s}$ in the form of strained amorphous regions. Thus, we do not expect this "block" length to affect the melting behavior to any serious extent. The "block" length should not be confused with $l_{s}$.

\subsection{The Apparent End Surface Free Energy.}

It is of interest to indicate the relationship of the bundleend surface free energy that we have found with theoretical estimates based on statistical mechanical treatments given in the literature. The cumulative strain-free portion of the end surface free energy in our formulation is $\sigma_{e 0}$; this result is

TABLE 2. Core crystallite dimensions as a function of flow field intensity: polyethylene in p-xylene.

\begin{tabular}{|c|c|c|c|c|c|c|c|}
\hline \multirow{2}{*}{$\begin{array}{c}\text { Crystallization } \\
\text { temperature } \\
\left({ }^{\circ} \mathrm{C}\right)\end{array}$} & \multirow{2}{*}{$\begin{array}{l}\text { Take-up } \\
\text { speed } \\
(\mathrm{cm} / \mathrm{min})\end{array}$} & \multicolumn{3}{|c|}{ Fibril diameter, $a_{s}(\AA)^{a}$} & \multicolumn{3}{|c|}{ Crystallite length, $l_{s}(\AA)^{b}$} \\
\hline & & DF & ED & WAXD & TEM & SAXS & $\begin{array}{c}\text { Axial Ratio } \\
l_{s} / a_{s}\end{array}$ \\
\hline 103 & 2 & 250 & 230 & 260 & 1500 & 1400 & 5.9 \\
\hline 103 & 8 & 200 & 210 & 210 & 1250 & 1300 & 6.2 \\
\hline
\end{tabular}

${ }^{a} \mathrm{DF}=$ dark field electron microscopy; $\mathrm{ED}=$ broadening of electron diffraction spots; WAXD = broadening of wide-angle $\mathrm{x}$-ray reflections. (Data of Zwijnenburg, et al $[20]$.)

${ }^{b}$ TEM $=$ transmission electron microscopy; SAXS = small angle x-ray scattering. Data from ref. [20] on distances between lamellar overgrowths. It is assumed here that these data represent the characteristic length $l_{s}$. 
new, and no previous theoretical estimates are available. However, some interesting formulations have been given, for instance by Zachmann [25, 26], for the bundle-end surface free energy using the assumption that none of the surface energy was transmitted to the lattice, i.e., the lattice dimensions were held fixed in the calculations. We refer to a bundle-end surface free energy calculated in this manner as $\sigma_{e}=\sigma_{e \text { (apparent). In the present formulation, it is found from }}$ eq (2l) together with $l / l_{c}=n_{s}$ and $\left(V / V_{c}\right)^{2 / 3}=n_{s}{ }^{2}$ that

$$
\begin{array}{r}
\sigma_{e(\text { apparent })}=\sigma_{e o}+\left(\nu \epsilon_{c}\right) n_{s}{ }^{3}=120.5+1325 \\
\cong 1445 \mathrm{erg} / \mathrm{cm}^{2} .
\end{array}
$$

The numerical value given refers to polyethylene, and was calculated using the data given in table 1 . It is this number that is to be compared with the theoretical calculations mentioned above that do not allow for lattice expansion and for some conversion of end surface free energy to volume strain. It would be of considerable interest to investigate using statistical mechanics the properties of models where volume strain is allowed, and where the bundle-end surface free energy exhibits two components - one of these being free of cumulative strain $\left(\sigma_{e 0}\right)$, and the other corresponding to the term $\left(\nu \epsilon_{c}\right) n_{s}{ }^{3}$ that derives from cumulative strain.

We have already noted the fact that annealing can cause $\sigma_{e}=\sigma_{e \text { (apparent) }}$ to fall to lower values $\left(\sim 550 \mathrm{erg} / \mathrm{cm}^{2}\right.$ for polyethylene) in such a manner as to cause the elimination of the volume strain. This will prove useful in the ensuing analysis of melting effects. For a case equivalent to no volume strain, Zachmann has estimated that $\sigma_{e} \sim 600 \mathrm{erg} /$ $\mathrm{cm}^{2}[25,26]$.

\subsection{Melting Phenomena: Independent Estimate of $\boldsymbol{l}_{\mathbf{s}}$.}

We consider first the shape of the melting curves. The overall objective in this part of the discussion is to find if the parameters that we have obtained are consistent with the shape of the crystallinity versus temperature curves of polyethylene fibrils with a reasonable value of $\bar{l}$. We begin by testing eqs (52) and (53a), which depend on $\sigma_{e 0}, \sigma$, and $\alpha n_{s}^{2}$, to determine if these parameters give a reasonable prediction of the shape of observed crystallinity $v s$ temperature curves with a value of $\bar{l}$ consistent with other estimates of this quantity. This particular test assumes that the crystallites are not annealed, so that the effect of volume strain has its full effect. This will give one bound for $\bar{l}$. However, it is reasonable to suspect from the method of preparation and the duration of the melting experiments that the volume strain in the fibrils may have relaxed. Therefore, we shall use eq (52) coupled with eq (53b) with $\sigma_{e}=550$ $\mathrm{erg} / \mathrm{cm}^{2}$ to fit the data to obtain another bound for $\bar{l}$.

In a paper concerned with crimping and melting-induced contraction of polyethylene fibrils that is of considerable general interest, Grubb and Keller measured the degree of crystallinity $\chi$ of fibrils in the absence of solvent as a function of temperature. It should be explicitly noted that the fibrils used by these authors were prepared by a different technique (solution stirring in xylene at $98{ }^{\circ} \mathrm{C}$ ) than that employed by George and Tucker. Grubb and Keller's data are depicted in figure 6 . The points above about $138{ }^{\circ} \mathrm{C}$ were stated to be subject to the effects of superheating (open circles), and will not be included in the analysis to follow. Their $\chi$ values were obtained from measurements of the

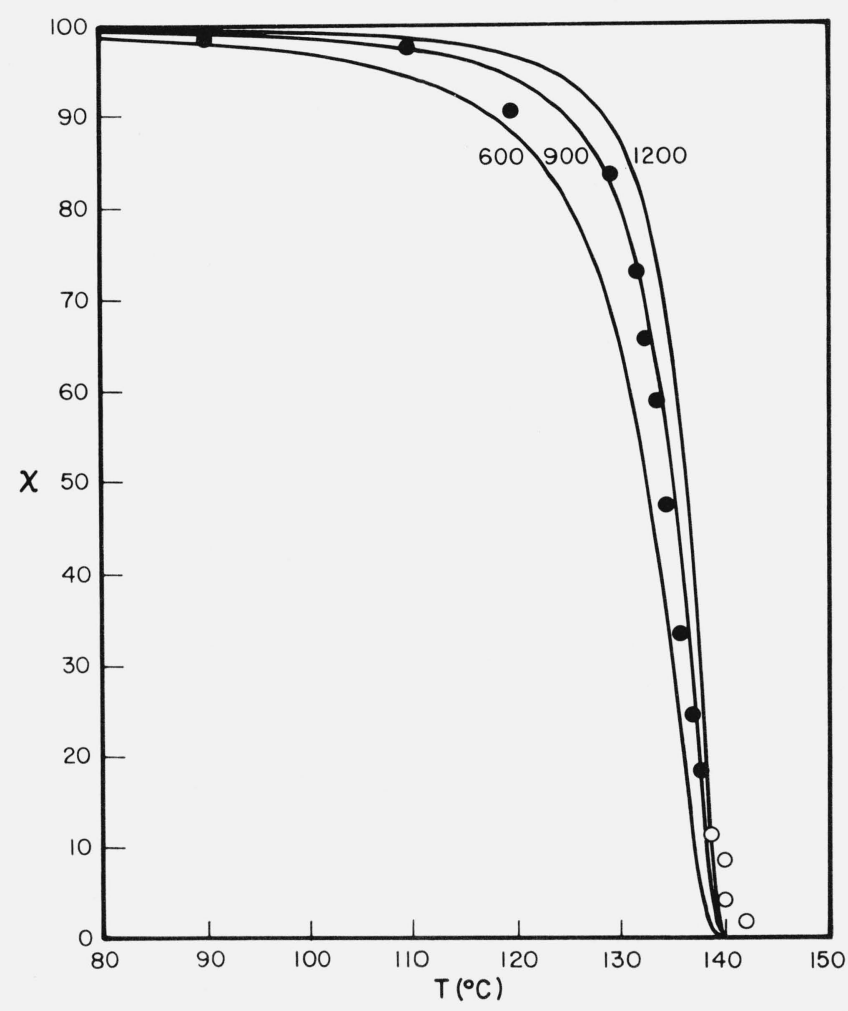

FIGURE 6. Degree of crystallinity from contraction measurements as a function of temperature for polyethylene core fibrils (effect of volume strain included).

Solid lines calculated for various $\bar{l}$ with eqs (52) and (53a) using input data from eq (62). Data of Grubb and Keller [11]: $(O)$ points affected by superheating or annealing (๑) points used in analysis. The best fit is obtained with $\bar{l} \cong 900 \AA$. This corresponds to a characteristic length of $\sim 1800 \AA$

overall length of the fibrils at various temperatures. Contraction occurs when an essentially extended-chain crystallite in the core fibril melts, the molecules in this region then being more random, thus shortening the fibril. The percentage of the contraction is a direct measure of the fraction of the core fibril that has melted. The shorter crystallites melt out first on warming, so that the shape of the $\chi$ versus $T$ plot reflects the distribution of crystallite lengths. The "crystallinity" calculated in this way does not deal with melting effects caused by the lamellar overgrowths, and does not include the small reduction in overall crystallinity caused by the amorphous regions. The reader is referred to Grubb and Keller's 
paper for further details of the measurements. We shall use the aforementioned $\chi$ versus $T$ data (other than those affected by superheating) to obtain a reasonable range of values of the characteristic length.

Attention is now drawn to the expression for $l_{o}$, eq (53a). In this expression, we already have from the input data in table 1 and the analysis of $a_{s}$ and $l_{s}$ given previously the following values:

$$
\begin{array}{ll}
\Delta h_{f}=2.8 \times 10^{9} \mathrm{erg} / \mathrm{cm}^{3} ; & \sigma=14.4 \mathrm{erg} / \mathrm{cm}^{2} \\
\alpha n_{s}^{2}=0.541 & \sigma_{e o}=120.5 \mathrm{erg} / \mathrm{cm}^{2}
\end{array}
$$

We consider that these numbers are accurate to within about 10 percent, and will not subject them to adjustment in the ensuing analysis. Eq (53a) also requires values of the mean diameter of the fibrils $a_{s}$, and the equilibrium melting temperature $T_{m}^{\circ}$.

$a_{s} \cong 300 \times 10^{-8} \mathrm{~cm} ; T_{m}{ }^{\circ}=146.5^{\circ} \mathrm{C}=419.7^{\circ} \mathrm{K}$

The value for $a_{s}$ is that suggested by Grubb and Keller for the fibrils used in their studies. No detailed statistics were given for this diameter, so we infer that this is actually a nominal value. The value of $T_{m}{ }^{\circ}$ is taken from an analysis in the literature [27] that is based at high molecular weights on the experimental studies of Rijke and Mandelkern [28], who found $T_{m}^{\circ}=146.0 \pm 0.5^{\circ} \mathrm{C}$ for fibrillar material. The latter experiments were of considerable time duration and designed in part for the specific purpose of eliminating the effects of superheating. Interesting long-term annealing effects were observed very near the melting point that were attributed by these authors to an increase of crystallite size and a possible reduction in the surface free energy.

The distribution of lengths in the particular type of samples studied by Grubb and Keller is evidently very broad, and they therefore used the distribution given in eq (46), which leads to eq (52) for $\chi$, in their analysis. We shall accordingly use eq (52) for calculations of $\chi$. Recall, however, that we utilize eq (53a), which contains $\alpha n_{s}^{2}$, to calculate $l_{o}$ in the present analysis. With these developments, the only parameter available for the fitting process is $\bar{l}$. In this process, the value of $\bar{l}$ tends to determine the general shape of the curve, while the ratio $\sigma / a_{s}$ determines the apparent intercept on the temperature coordinate as $\chi$ $\rightarrow 0$.

The results of the calculations with eqs (52) and (53a) with the input data noted above, and $\bar{l}$ values of 600,900 , and $1200 \AA$ are shown in figure 6 . In the region where superheating does not interfere, a good fit is obtained with $\bar{l} \cong 900$ $\AA$. There is an interesting check on the validity of this number, since it leads through eq (47a) to a mean value of $l$ of $1800 \AA$. This value represents an independent estimate of the magnitude of $l_{s}$ (calculated as $\langle l\rangle=2 \bar{l}$ ) that does not depend on measurements of the lamellar overgrowth spacing. Remembering that $a_{s} \cong 300 \AA$, this corresponds to a mean axial ratio of $\gamma=1800 / 300=6$, which is in rough agreement with that found from our analysis of the data of George and Tucker, and quite close to that we estimate from the data of Zwijnenburg et al [20] (see Sec. 4.3). Thus in the case where volume strain is assumed to be present, the melting data imply a reasonable value of $\bar{l}$ and $\bar{l}_{s}$.

We now assume that the volume strain has relaxed in the fibrils studied by Grubb and Keller, and utilize eq (52) with eq $(53 \mathrm{~b})$ to analyze the melting data. The value $\sigma_{e}=550$ $\mathrm{erg} / \mathrm{cm}^{2}$ is used in eq (53a) for the reasons cited in section 4.4. The other input data are given in eqs (62). A reasonable fit is obtained with $\bar{l}=1200 \AA$ (not shown). The degree of fit is comparable to that achieved by Grubb and Keller, and tends to confirm their original analysis with $\sigma_{e}=600 \mathrm{erg} /$ $\mathrm{cm}^{2}$, this surface free energy having been obtained from Zachmann's calculation. The present analysis gives $l_{s}=2 \vec{l}$ $=2400 \AA$, which leads to a mean axial ratio of $2400 / 300=$ 8 , which is satisfactory.

While it is not possible to decide which of the two estimates of $\bar{l}$ given above is more nearly correct, since one cannot be certain whether or not the volume strain in the specimens was relaxed, the results are still highly significant in a general way. They show that $\bar{l}$ is very probably in the range of $900 \AA$ to $1200 \AA$. This implies that the average distance between full "amorphous" interruptions of the core fibrils studied was 1800 to $2400 \AA$. Despite certain differences, we regard this analysis as generally confirmatory of the model proposed by Grubb and Keller, as well as of our contention based on the present theoretical development that the core fibril is interrupted every few thousand $\AA$ by an amorphous region.

The present analysis tends to further verify that the function, eq (46), suggested by Grubb and Keller to describe the distribution of the spacing of the amorphous regions along the core fibril is suitable for material produced in the particular manner (stirrer technique) used by them. It would be of considerable interest to determine if this distribution function applied to the melting curves of fibrils produced by the method employed by George and Tucker. The data of these authors suggest that the distribution about $l_{s}$ may be more narrow than that given in eq (46), and implies further that the distribution is approximately Gaussian (compare CV values in table 1 with eq $(47 \mathrm{c})$ ). The theory given in this paper implies that considerably more narrow distributions than that displayed as eq (46) are possible in suitably prepared specimens.

The mean distance between the amorphous regions as determined from melting curve data seems to be limited as required by the theory, and exhibit an absolute value of the correct magnitude. The mean value of the lamellar overgrowth spacings obtained for a core fibril $300 \AA$ A thick formed 
by the technique employed by George and Tucker is in at least rough agreement with an independent estimate of the mean crystallite length for a fibril formed by a rather different technique, but with nominally the same diameter. As a corollary, there appears to be no compelling reason to reject the hypothesis that there exists an approximate correspondence between the mean value of the characteristic crystallite length $l_{s}$ and the mean value of the distance between lamellar overgrowths when the latter is obtained from material produced according to the experimental scheme used by George and Tucker. The converse is not necessarily true; solution stirred fibrils could easily possess extra overgrowths that might lead to low estimates of $l_{s}$. One must also be aware of the possibility that the distribution in $l$ may depend on the mode of preparation and annealing of the fibrils.

It is emphasized that the type of "defect" involved in the discussion of melting phenomena is a fully developed strained amorphous region that interrupts the crystalline part of the core fibril. The distribution of such gross defects determines the shape of the melting curves. "Partial" amorphous defects, where the body of the core fibril is not interrupted, will have a considerably smaller effect. Defects of the usual type (e.g., kinks, dislocations, and structural anomalies associated with chain ends and folds) may occur in the crystallites, but these, even though numerous, cannot be expected to alter the melting behavior to anything like the extent as do the presence of the amorphous regions and the finite diameter of the fibrils. It is clear that the "blocks" of length 250 to $300 \AA$ mentioned in section 5.3 are not the principal cause of the melting phenomena treated here or by Grubb and Keller.

We now note the connection that may exist between certain aspects of the treatment given here, and processes that may allow a close approach of the melting of a fibrillar system to the equilibrium melting temperature, $T_{m}{ }^{\circ}$.

The melting point depression of a polyethylene fibril 300 $\AA$ thick with cumulative strain is readily calculated from eq (50) to be $9.4{ }^{\circ} \mathrm{C}$. If the amorphous zones were somehow removed and the volume strain relaxed by some annealing process, then the melting point depression for a $300 \AA$ fibril calculated with eq (48) with $l \rightarrow \infty$ or eq $(51)$ is only $2.9^{\circ} \mathrm{C}$. It is reasonable to suppose that some such process took place during the prolonged storage near $T_{m}{ }^{\circ}$ in the experiments on polyethylene fibrils carried out by Rijke and Mandelkern [28], in which they closely approached $T_{m}^{\circ}$. They attributed the high melting point $\left(146.0{ }^{\circ} \mathrm{C}\right)$ that they achieved after prolonged storage at $142{ }^{\circ} \mathrm{C}$ to either an increase of crystal size, or a reduction in surface free energy. We are in general agreement with this statement, and suggest the following more detailed interpretation. The attainment of a melting point so close to the estimated value of $T_{m}{ }^{\circ}$ by these investigators for fibrillar material by prolonged high temperature annealing strongly implies that the amorphous zones in the core fibril can be removed. Specifically, we view this increase in melting point as mainly a result of an increase of molecular mobility, which allows diffusive removal of the amorphous zones that in turn eliminates the end surface free energy and volume strain effects on the melting behavior. (These results also imply that the minimum that we have predicted at $n_{s}$ should be regarded as metastable in character.) Although Grubb and Keller attribute the high melting points obtained at low crystallinities (open circles in fig. 6) to the effects of superheating of the core fibrils, it seems possible that some of this high melting effect may have been a result of the annealing processes discussed above.

An increase in the mean value of the crystallite length, and possibly in the shape of the distribution function, should accompany the high temperature annealing process.. Also, the corresponding reduction in volume strain will tend to remove the thermodynamic restriction on an increase of $a_{s}$ (fig. 2C), and this may eventually allow mobile polymer chains arising from melted out lamellar overgrowths or adsorbed molecules to slowly increase the diameter of the fibril. In any case, a sufficiently annealed core fibril may have few overgrowths, and only a few amorphous regions, but some adsorbed molecules would still be present.

\subsection{Lattice Expansion in the Core Fibril Caused by Cumulative Strain.}

George and Tucker have given electron diffraction data obtained at room temperature on the thinner $(181 \AA)$ of the two fibrils mentioned in table 1 , i.e., that formed at $84{ }^{\circ} \mathrm{C}$. They were easily able to identify not only the (110) and (200) spacings, but also many others such as (002), (004), (210), (310), (311), and the (401) reflections. They make a definite statement that the lattice is considerably expanded, and quote the (110) and (200) spacings (table 3). The lattice is orthorhombic. Further, they cite evidence that this expansion was not the result of electron beam damage. The experiments were repeatable, and non-fibrillar samples showed no large lattice expansion [29]. The electron diffraction data appear to give reflections that are consistent with an approximately uniform expansion of the lattice, though some smearing of some of the spots was apparent [29]. ${ }^{8}$ By using the precise $x-$ ray data of Davis et al as a base line for the cross-sectional area of the infinite crystal, i.e., unstrained lattice, it is readily determined that the lattice expansion for the $181 \AA$ diameter core fibril was $0.86 \AA^{2}$ or 4.7 percent (table 3 ). There is undoubtedly a rather large experimental uncertainty associated with this value because of the errors inherent in

${ }^{8}$ The model we have suggested for the cause of the overall lattice expansion (small chain translations resulting from fluctuations in the local tensions caused by ciliary bridges of varying size and conditions) allows for some local inhomogeneity which might contribute to smearing. Also, there may be some difference in the volume strain in the central core as compared to the regions near the outer surface, as well as differences in volume strain in the central part of the core crystallite as compared to that near the amorphous zones. 
TABLE 3. Effect of cumulative volume strain on cross-sectional area of chains in polyethylene fibrils.

\begin{tabular}{|c|c|c|}
\hline \multicolumn{3}{|c|}{ A. Input Data } \\
\hline Quantity & Value & Remarks \\
\hline $\begin{array}{l}\text { 1. Cross-sectional area of chains with no volume } \\
\text { strain, } A_{o}\end{array}$ & $18.23 \AA^{2}\left(23^{\circ} \mathrm{C}\right)$ & Davis et al, ref. [16] \\
\hline $\begin{array}{l}\text { 2. Cross-sectional area of chains in core fibril } 181 \AA \\
\text { in diameter, } A_{o} \text { (strain) }\end{array}$ & $19.09 \AA^{2}$ (room temp.) & $\begin{array}{l}\text { George and Tucker, ref. [12] Calc. from (110) spacing }=4.2 \AA \text {, } \\
\text { (200) spacing }=3.78 \AA\end{array}$ \\
\hline \multirow{2}{*}{$\begin{array}{l}\text { 3. } K_{1} \text { in eq }(55) \\
\text { 4. } K_{2} \text { in eq }(56)\end{array}$} & $160 \mathrm{Jg}^{-1} \AA\left(23^{\circ} \mathrm{C}\right)$ & \multirow{2}{*}{$\begin{array}{l}\text { Davis et al, ref. [15] (Interpolated from their figure 9.) } \\
\text { Davis et al, ref. [16]. Average for solution and melt-crystallized } \\
\text { polymer. Valid for chain-folded systems. }\end{array}$} \\
\hline & $15.7 \pm 3 \AA^{3}\left(23^{\circ} \mathrm{C}\right)$ & \\
\hline 5. $K_{2}$ in eq $(57)$ & $20.3 \AA^{3}\left(23^{\circ} \mathrm{C}\right)$ & \multirow{2}{*}{$\begin{array}{l}K_{2} \text { in (4) above corrected by factor } \sigma_{e o} / \sigma_{e(\text { fold })}=120.5 / 93= \\
1.30 . \text { Valid for bundlelike systems. } \\
\text { Valid for bundlelike systems. }\end{array}$} \\
\hline 6. $K_{2} / K_{1}$ in eq $(57)$ & $0.127 \mathrm{Jg}^{-1} \AA^{2}$ & \\
\hline \multicolumn{3}{|c|}{ B. Results } \\
\hline \multirow{2}{*}{ Fibril Description } & \multicolumn{2}{|c|}{ Lattice Expansion in Core Fibril } \\
\hline & Exptl. [12] & Theor., eq (57) \\
\hline $\begin{array}{l}a_{s}=181 \AA \\
a_{s}=303 \AA\end{array}$ & $0.86 \AA^{2}$ or $4.7 \%$ & $\begin{array}{l}0.71 \AA^{2} \text { or } 3.9 \% \\
0.44 \AA^{2} \text { or } 2.4 \%\end{array}$ \\
\hline
\end{tabular}

the electron diffraction experiments, but there is little doubt from an experimental standpoint as regards the existence of an expansion of at least a few percent.

The theoretical estimate of the lattice expansion with eq (57) is straightforward. The details are given in table 3 . Observe that we have corrected $K_{2}$ by the use of the factor $\sigma_{e o} / \sigma_{\text {(fold) }}=1.30 \mathrm{in}$ order to more realistically represent this parameter for a bundlelike system. Even without this correction, a large expansion is predicted. A density of 1.00 $\mathrm{g} / \mathrm{cm}^{3}$ was used in the calculations.

It is seen in table 3 that the predicted increase in crosssectional area for the $181 \AA$ fibril is $0.71 \AA^{2}$ or 3.9 percent. Considering the possible experimental errors, and our earlier remarks concerning the assumptions inherent in the theory, the extent of agreement evident in table 3 may be regarded as satisfactory. Note that the thicker fibril, $a_{s}=303 \AA$, is predicted to have a smaller degree of expansion. No experimental values for the degree of expansion are available for this specimen. It is probably significant that the lattice expansion effect appears clearly in a specimen formed rapidly, and at a temperature well below that where thermally activated translational motions of the chains are present (see remarks in sec. 4.4). Under these circumstances, annealing of the volume strain is less likely to occur. There is no information on whether or not the fibrils exhibiting the expanded lattice were in a state of mechanical tension. If present, this may have played a role in stabilizing the state with the expanded lattice (sec. 4.4). From our understanding of the experimental technique utilized by George and Tucker, we consider that it is entirely possible that the individual fibrils were pinned at various points onto the evaporated carbon substrate, in which case it is probable that they were in a state of tension characteristic of their actual condition of formation.
The remarkable character of the lattice expansion exhibited by these unannealed polyethylene fibrils is afforded by a comparison with that found for thin chain folded crystals, where cumulative strain does not occur. According to eq (56), a folded crystal $100 \AA$ thick has an expansion of only 0.86 percent, and for thicker single crystals or lamellae it is even smaller. ${ }^{9}$

The foregoing analysis shows that an unannealed polyethylene core fibril can exhibit considerable volume strain, and supports our view that this strain is the natural result of repulsions originating at the bundle ends. The agreement achieved by the theoretical analysis suggests that the particular cumulative strain approximation used here is adequate in the present application.

\subsection{Remarks Concerning Lamellar Overgrowths.}

Although the discussion must necessarily be rather speculative, the present theory of core fibril formation may provide a background for improved understanding of the lamellar overgrowths ("kabobs") and their behavior.

It is first emphasized that a direct connection between the amorphous regions in the core and the position of the lamellar overgrowths has not been demonstrated in the present analysis. However, it has been shown for certain preparations that this is a reasonable postulate, and we shall proceed on this basis. Some conditions under which the lamellar overgrowths are most likely to mark the amorphous

\footnotetext{
${ }^{9}$ It is of interest to note that the electron diffraction data of George and Tucker on non-fibrillar specimens exhibited lattice expansions close to 1.0 percent [29], which is close to that predicted by eq (56). This adds further weight to the validity of their results on fibrillar material, where larger lattice expansions were found, since it implies that beam damage or some other artifact did not seriously affect the experimental technique.
} 
zones in the core fibril have been noted earlier. Below these concepts will be extended.

In a fresh preparation where the volume strain still exists in the core fibril crystallites, the outer parts of the strained amorphous zones (and any cilia that may be present) may actually comprise the interior portion of the lamellar overgrowth. In this sense, the interior part of the overgrowth may be an integral part of the core fibril. In brief, the bulge at one of the amorphous zones depicted in figure $1 \mathrm{C}$ may actually constitute the inner zone of the corresponding lamellar overgrowth (not shown). Such a condition could persist even after the first stage of annealing where the crystallite lattice first reverts to its unexpanded state.

As suggested earlier, the amorphous zones may diffuse out of the core fibril on full annealing (or preparation) at high temperatures. In such a case, the overgrowths would tend to disappear, leaving behind a smooth core fibril with only a few widely spaced amorphous interruptions. (It is well known that smooth fibrils can be formed at high crystallization temperatures.) Such a fibril should exhibit a higher melting point and considerably sharper melting behavior than an unannealed one with more numerous amorphous interruptions. The observation of such effects would support the view that there is a connection between overgrowths and the amorphous zones.

Consider a fibril from which the amorphous zones (and hence the overgrowths) have been mostly removed by high temperature preparation or prolonged annealing. We would postulate that such a core fibril would have a considerable number of polymer molecules physically adsorbed on its surface. ${ }^{10}$ These would be exceedingly difficult to remove $[23,30]$, though transport across the lateral surfaces would readily occur. On cooling, the adsorbed molecules could form chain-folded overgrowths consistent with the presence of a constant mass of overgrowth material per unit length of core crystallite. These overgrowths should tend to peak at the few widely spaced amorphous zones, but other sites (e.g., "blocks") might well compete for the adsorbed molecules because of various factors, including the large distance between the amorphous regions, and the less well-defined character of the amorphous zones resulting from annealing. The concepts noted here may in part prove useful in interpreting some of the observations of Pennings, Lagaveen, and Vries [13] on annealed smooth core fibrils that were cooled in solution to form lamellar overgrowths whose spacings were highly dependent on the storage temperature. In the light of the foregoing discussion, it is seen that the interesting phenomena observed by these investigators need not necessarily be construed as being antithetical to the concept that the lamellar overgrowths denote the site of the amorphous zones in the core fibril in fresh preparations

10 The author is indebted to Professor Andrew Keller for a very helpful discussion concerning this point. where suitable steps have been taken to minimize rearrangements and additional overgrowths.

While no completely firm conclusions can be drawn, it is nevertheless evident that the concept that the lamellar overgrowths denote the position of the amorphous zones in the core fibril in certain reasonably well-defined situations is consistent with a considerable body of information that is presently available. Interlamellar spacing data that were believed to fall in this category were used to estimate $l_{s}$ in the present paper. Meanwhile, it is clear that quite different but also fairly well defined circumstances exist where additional overgrowths can be caused to form on the fibrils, and still others where the overgrowths can change their spacings on storage.

\section{Overview}

We consider here some general points. The principal results and conclusions have been given earlier, and need not be repeated.

It is of interest to contrast the cause of the limitation of crystallite dimensions that occurs in the case of crystallization with chain folding with that which occurs in the bundlelike core fibril. The discussion is confined to the case of solutions.

Crystallization with chain folding occurs from more or less quiescent solutions where the polymer molecules possess little, if any, net orientation. An individual single crystal can grow to very large dimensions in the direction transverse to the chain axes. There is no evidence of any fundamental limitation to growth in this direction. The folds fit together in such a way as to obviate cumulative strain, though small non-cumulative volume strain effects do occur. The surface free energy of the fold surface, $\sigma_{e(f o l d)}$, is comprised principally of the term $q / 2 A_{o}$ where $q$ is the work of chain folding; $\sigma_{e(\text { fold })}$ is independent of the size of the crystal. The fold period (before any thickening takes place) is determined by strictly kinetic factors; a thermodynamic theory of the initial fold period is inappropriate. The flux across the nucleation barrier system is a sensitive function of the thickness of a chain-folded embryo attempting a transit into the stable region. This flux is proportional to $\exp \left[-4 b_{0} \sigma \sigma_{e(\text { fold })} /(\Delta f) k T\right]$, where $b_{o}$ is the molecular layer thickness, and appropriate averages utilizing this function give both the growth rate and the initial fold period associated with a specified undercooling [23]. The fold period is given by $l_{g}{ }^{*}=2 \sigma_{e(\text { fold })} /(\Delta f)+$ $\delta l$, where $\delta l$ may be regarded as a constant for the purposes of this discussion. At any given undercooling, crystals that are significantly thicker or thinner than $l_{g}{ }^{*}$ cannot surmount the nucleation barrier system, but crystals with a thickness close to $l_{g}{ }^{*}$ surmount the barrier, enter the stable region, and grow in the direction transverse to the chain axes without further restrictions; there is no minimum corresponding to $n_{s}$ 
in the stable region. Thus, nucleation kinetics and the absence of cumulative strain dominate both the rate of growth and the crystal dimensions of chain-folded crystals in dilute solutions [23].

Consider now the case of fibril formation in solution. This phenomenon only occurs in the case of polymer molecules that are statistically elongated by a flow field. The system is committed at the outset to a basically fringed-micellar mode of crystallization largely because of multiple nucleation acts of elongated molecules on a central elongated molecule, or embryonic fibril core (fig. 1A). This leads to a set of nuclei or crystallites of the extended-chain type connected together by amorphous regions. The system must now bear the consequences of the presence of these quasi-random coil amorphous regions as the nuclei attempt to grow. According to the present theory, the crystallites in the core fibril have a fundamental limitation on their dimensions of a thermodynamic character. The primary cause of this limitation is the mutual repulsion between the excessive number of ciliary bridges that emanate from the bundle ends and then re-enter an adjacent crystallite. These repulsions are small as the embryo slowly forms, because time permits folds and short cilia and possibly other defects to enter the bundle end and mitigate these repulsions. The flow field produces elongated molecules at or near the crystallization sites. When very rapid growth begins at or near $V_{c}$, i.e., in the stable region, these aligned molecules are forcibly incorporated into the crystallite, producing a bundlelike end surface with an excessive fraction of ciliary bridges that exhibit increasingly strong repulsions, and cumulative strain begins to predominate. (Observe that it is the size of a nucleus and the rate at which it forms that determines where cumulative strain becomes important. The volume $V_{c}$ is defined as $a_{c}^{2} l_{c}$ where $a_{c} \propto \sigma /(\Delta T)$ and $l_{e} \propto \sigma_{e o} /(\Delta T)$; the final "stable" dimensions of the crystallite, $a_{s}$ and $l_{s}$, reflect these relationships, though they are much larger. Thus, kinetics play a subtle but definite role, even though the final limitation that prevails is basically thermodynamic in character.) The situation just described leads directly to cumulative surface stress at the bundle ends as the area of the crystallite ends increase. This becomes so large that some of the energy is transmitted by the agency of chain translation to the body of the crystallite, which expands its lattice, and increases its free energy. These processes create a minimum in the total free energy of the crystallite, which leads to a definite limitation on each of its dimensions. (Annealing mechanisms evidently exist whereby the volume strain can be relaxed, and prolonged storage at high temperatures can largely eliminate the amorphous zones: accordingly this minimum is best described as being metastable.) Both the metastable diameter $a_{s}$ and the metastable crystallite length $l_{s}$ vary as the reciprocal of the undercooling. The ratio of these dimensions is $l_{s} / a_{s}=\sigma_{e d}$ $\sigma$, and the absolute value of $a_{s}$ and $l_{s}$ depends on a parameter $\alpha$ that is related to the rate the cumulative strain builds up as the volume and length of the core crystallites increase. Thus, the bundlelike crystallite in the core fibril begins its life slowly and essentially free of strain, but upon reaching a state of being just stable, begins a period of rapid and improvident growth that causes it to collect and retain defects in the form of ciliary bridges emanating from the bundle ends that in an ordinary time scale limit both its mean length and diameter.

The author is particularly grateful to Professors Waller George and Paul Tucker for numerous helpful discussions concerning the details of their experiments, and for their comments on the draft manuscript. Special thanks are also due Professors Andrew Keller and David Grubb for making their work on the contractility of polyethylene fibers known to the author well in advance of its publication, and for their most useful comments. We also wish to thank Professors R. J. Gaylord, S. H. Carr, G. Yeh, and Dr. R. L. Miller, for their critique of the manuscript. Finally, the author wishes to thank NBS staff members including Drs. J. W. Cahn, J. T. Fong, C. A. Guttman, and E. A. DiMarzio for many fruitful discussions.

\section{Appendix}

\subsection{Alternative derivation of eq (28).}

Write the free energy of formation as

$$
\Delta \phi=2 a^{2} \sigma_{e o}+4 a l \sigma-a^{2} l(\Delta f)+V_{d} \epsilon \lambda
$$

where $V_{d} \epsilon \lambda=$ volume strain free energy in ergs, $V_{d}=$ volume in which cumulative strain takes place in $\mathrm{cm}^{3}, \epsilon=$ erg/cilium or ciliary bridge as before, and $\lambda=$ number of cilia or ciliary bridges per $\mathrm{cm}^{3}$ of crystallite. Now we let

$$
V_{d}=V-V_{c} .
$$

Also,

$$
\lambda=\frac{\text { number of cilia on bundle ends }}{\text { volume of crystal }}=\frac{2 \nu a^{2}}{a^{2} l}=\frac{2 \nu}{l}
$$

where $\nu=$ number of cilia on bundle ends per $\mathrm{cm}^{2}$, so that

$$
V_{d} \epsilon \lambda=2 \nu \epsilon\left(V-V_{c}\right) / l .
$$

Then with $\nu \epsilon=\nu \epsilon_{c}\left(l / l_{c}\right)\left(V / V_{c}\right)^{2 / 3}, l_{c}=6 \sigma_{e o} /(\Delta f)$ and $\alpha \equiv$ $\nu \epsilon_{c} / 3 \sigma_{e o}$, one gets

$$
\begin{aligned}
\Delta \phi=2 a^{2} \sigma_{e o}+4 a l \sigma- & a^{2} l(\Delta f) \\
& +\alpha(\Delta f)\left(V / V_{c}\right)^{2 / 3}\left(V-V_{c}\right) .
\end{aligned}
$$

Substitution of eqs (27) into (A-5) gives eq (28). 


\section{References}

[1] Pennings, A. J. and Kiel, A. M. Kolloid-Z., 205, 160 (1965).

[2] Pennings, A. J. J. Polymer Sci., C-16, 1799 (1967).

[3] Pennings, A. J. van der Mark, J. M. A. A. and Booij, H. C. KolloidZ., 236, 99 (1970).

[4] Pennings, A. J. and Pijpers, M. F. J. Macromolecules, 3, 261 (1970).

[5] Pennings, A. J. van der Mark, J. M. A. A. and Kiel, A. M. KolloidZ., 237, 336 (1970).

[6] Pennings, A. J. J. Polymer Sci., Polymer Symposium No. 59, 55 (1977), (J. Wiley and Sons, New York).

[7] Zwijnenburg, A. and Pennings, A. J. Colloid and Polymer Sci., 253, 452 (1975).

[8] Pennings, A. J. Zwijnenburg, A. and Lagaveen, R. Kolloid Z. u. Z. Polymere, 251, 500 (1973).

[9] Mackley, M. R. and Keller, A. Polymer, 14, 16 (1973).

[10] Mackley, M. R. Colloid and Polymer Sci., 253, 373 (1975).

[11] Grubb, D. T. and Keller, A. Colloid and Polymer Sci., 256, 218 (1978).

[12] George, W. and Tucker, P. Polymer Eng. and Sci., 15, 451 (1975).

[13] Pennings, A. J. Lageveen, R. and Vries, R. S. Colloid and Polymer Sci., 255, 532 (1977).

[14] Fong, J. T. J. Res. Nat. Bur. Stand. (U.S.), to be published.

[15] Davis, G. T. Eby, R. K. and Colson, J. P. J. Appl. Phys., 41, 4316 (1970).

[16] Davis, G. T. Weeks, J. J. Martin, G. M. and Eby, R. K. J. Appl. Phys., 45, 4175 (1974).
[17] Bolz, L. H. and Eby, R. K. Inclusion of perfluoromethyl groups in the crystals of copolymers of tetrafluoroethylene and hexafluoropropylene, J. Res. Nat. Bur. Stand. (U.S.), 69A (Phys. and Chem.), No. 5, 481-486 (Sept.-Oct. 1965).

[18] DiMarzio, E. A. Bull. Am. Phys. Soc., 23, No. 3, 420 (1978).

[19] Flory, P. J. J. Am. Chem. Soc., 84, 2857 (1962).

[20] Zwijnenburg, A. van Hutten, P. F. Pennings, A. J. and Chanzy, H. D. Colloid and Polymer Sci. 256, 50 (1978).

[21] Andersen, P. G. and Carr, S. H. Polymer Eng. and Sci. 18, 215 (1978).

[22] Huseby, T. W. and Bair, H. E. J. Appl. Phys., 39, 4969 (1968).

[23] Hoffman, J. D. Davis, G. T. and Lauritzen, Jr., J. I. Treatise on Solid State Chemistry, N. B. Hannay, ed., Vol. 3, Chapter 7, pp. 497614, (Plenum Press, 1976).

[24] Hoffman, J. D. Williams, G. and Passaglia, E. J. Polymer Sci. C-14, 173 (1966).

[25] Zachmann, H. G. Kolloid Z. u. Z. Polymere, 216, 180 (1967).

[26] Zachmann, Kolloid Z. u. Z. Polymere, 231, 504 (1969).

[27] Hoffman, J. D. Ross, G. S. Frolen, L. and Lauritzen, Jr., J. I. On the growth rate of spherulites and axialites from the melt in polyethylene fractions: Regime I and regime II crystallization, J. Res. Nat. Bur. Stand, (U.S.), 79A (Phys. and Chem.), No. 6, 671-699 (Nov.Dec. 1975).

[28] Rijke A. M. and Mandelkern, L. J. Polymer Sci., A-2, 8, 225 (1970).

[29] Tucker, P. and George, W. private communication.

[30] Hoffman, J. D. and Davis, G. T. On the origin of the amorphous component in polymer single crystals and the nature of the fold structure, J. Res. Nat. Bur. Stand. (U.S.), 79A (Phys. and Chem.) 613-617, No. 5 (Sept.-Oct. 1975). 